\title{
The distance between the two BBM leaders
}

\author{
Julien Berestycki* Éric Brunet ${ }^{\dagger} \quad$ Cole Graham $^{\ddagger} \quad$ Leonid Mytnik ${ }^{\S}$ \\ Jean-Michel Roquejoffre $\mathbb{I}^{\text {Lenya Ryzhik }} \|$
}

October 21, 2020

\begin{abstract}
We study the distance between the two rightmost particles in branching Brownian motion. Derrida and the second author have shown that the long-time limit $d_{12}$ of this random variable can be expressed in terms of PDEs related to the Fisher-KPP equation. We use such a representation to determine the sharp asymptotics of $\mathbb{P}\left(d_{12}>a\right)$ as $a \rightarrow+\infty$. These tail asymptotics were previously known to "exponential order;" we discover an algebraic correction to this behavior.
\end{abstract}

\section{Introduction}

We consider a branching Brownian motion (BBM) that starts with a single particle at the position $x=0$ at time $t=0$, with branching occurring at rate 1 . At each branching event, the particle splits into a random number of particles. Let $p_{k}$ denote the probability of $k$ offspring in a given branching event. We assume that $p_{0}=p_{1}=0$, so

$$
\sum_{k=2}^{\infty} p_{k}=1
$$

We let $N$ denote the expected number of offspring:

$$
N=\sum_{k=2}^{\infty} k p_{k}<\infty
$$

and we assume that the offspring distribution has a higher moment:

$$
\sum_{k=2}^{\infty} k^{1+\beta} p_{k}<\infty \quad \text { for some } \beta \in(0,1) .
$$

\footnotetext{
*Department of Statistics and Magdalen College, University of Oxford, UK; julien.berestycki@stats.ox.ac.uk

${ }^{\dagger}$ Laboratoire de Physique de l'École normale supérieure, ENS, Université PSL, CNRS, Sorbonne Université, Université de Paris, F-75005 Paris, France; eric.brunet@ens.fr

${ }^{\ddagger}$ Department of Mathematics, Stanford University, Stanford, CA 94350, USA; grahamca@stanford.edu

${ }^{\S}$ Faculty of Industrial Engineering and Management, Technion, Technion City, Haifa 3200003, Israel; leonid@ie.technion.ac.il

${ }^{\mathbb{I}}$ Institut de Mathématiques de Toulouse; UMR 5219, Université de Toulouse; CNRS, UPS IMT, F-31062 Toulouse Cedex 9, France; E-mail: Jean-Michel.Roquejoffre@math.univ-toulouse.fr

"Department of Mathematics, Stanford University, Stanford, CA 94350, USA; ryzhik@stanford.edu
} 
To simplify the diffusion constant in the Fisher-KPP equation below, we assume that the variance of each individual Brownian motion is $2 t$ (rather than $t$ ). Let $x_{1}(t) \geq x_{2}(t) \geq \ldots \geq x_{n(t)}$ denote the positions of the particles that exist at time $t \geq 0$. Here, $n(t)$ is the total number of particles present at time $t$. Note that $n(t)$ is itself random. It is well known from the work of Bramson in [4, 5], followed by the paper by Lalley and Sellke [13], with a shorter proof in a more recent paper by Roberts [19], that the maximal particle $x_{1}(t)$ is typically at distance $O(1)$ from the position

$$
m(t)=c_{*} t-\frac{3}{2 \lambda_{*}} \log (t+1)
$$

as $t \rightarrow \infty$, with

$$
c_{*}=2 \sqrt{N-1}, \quad \lambda_{*}=\sqrt{N-1} .
$$

This result does not depend on the precise nature of branching, so that both the spreading speed $c_{*}$ and the logarithmic correction in (1.3) depend only on the expected number of offspring $N$.

To see the connection between Bramson's results and PDEs, recall that McKean showed in [15] that the cumulative distribution function of the maximal particle

$$
H(t, x)=\mathbb{P}\left[x_{1}(t) \geq x\right]
$$

satisfies the Fisher-KPP equation [9, 12]

$$
\partial_{t} H=\partial_{x}^{2} H+f(H),
$$

with the initial condition

$$
H(0, x)=\mathbb{1}_{(-\infty, 0]}(x) .
$$

The nonlinear reaction in (1.6) has the form

$$
f(u)=1-u-\sum_{k=2}^{\infty} p_{k}(1-u)^{k} .
$$

Bramson's probabilistic results imply that there is a constant $\bar{x}_{0} \in \mathbb{R}$ such that

$$
H(t, x+m(t)) \rightarrow U\left(x-\bar{x}_{0}\right) \quad \text { as } t \rightarrow+\infty
$$

uniformly on $\mathbb{R}$. Here, $U(x)$ is a Fisher-KPP traveling wave, so that $U\left(x-c_{*} t\right)$ is a solution to (1.6) moving with speed $c_{*}$. The function $U(x)$ satisfies

$$
-c_{*} U^{\prime}=U^{\prime \prime}+f(U), \quad U(-\infty)=1, \quad U(+\infty)=0
$$

and has the asymptotics

$$
\begin{array}{ll}
U(x) \sim x e^{-\lambda_{*} x} & \text { as } x \rightarrow+\infty, \\
U(x) \sim 1-A e^{\gamma_{*} x} & \text { as } x \rightarrow-\infty
\end{array}
$$

for

$$
\gamma_{*}=\sqrt{N}-\sqrt{N-1}
$$

and some $A>0$. Any translate of a solution to (1.10) is also a solution. However, there is a unique shift such that the pre-factor in front of the right side of (1.11) is one. This, in turn, identifies the constant $A$.

More generally, we have the following PDE result originating in work of Bramson $[4,5]$ and Uchiyama [20] and later developed in $[10,11,14,17,18]$. Let $u(t, x)$ solve $(1.6)$,

$$
\partial_{t} u=\partial_{x}^{2} u+f(u)
$$


with initial condition

$$
u(0, x)=\phi(x)
$$

for a non-negative bounded function $\phi \not \equiv 0$ which is compactly supported on the right. That is, there exists $L_{0}>0$ so that $\phi(x)=0$ for all $x \geq L_{0}$. Assume that $f \in \mathcal{C}^{1, \beta}([0,1])$ satisfies the Fisher-KPP assumptions

$$
f(0)=f(1)=0,\left.\quad f\right|_{(0,1)}>0, \quad \text { and } \quad f(u) \leq f^{\prime}(0) u \text { for all } u \in(0,1) .
$$

Then, there exists a constant $s[\phi] \in \mathbb{R}$ (depending also on $f$ ), known as the Bramson shift corresponding to $\phi$, such that

$$
u(t, x+m(t)) \rightarrow U(x-s[\phi]) \quad \text { as } t \rightarrow+\infty
$$

uniformly on compact sets, with $c_{*}=2 \sqrt{f^{\prime}(0)}$ and $\lambda_{*}=\sqrt{f^{\prime}(0)}$ in the definition (1.3) of $m(t)$. In particular, (1.9) states that

$$
s\left[\mathbb{1}_{(-\infty, 0]}\right]=\bar{x}_{0} .
$$

It is straightforward to check that $f(u)$ defined in (1.8) satisfies the Fisher-KPP property (1.13). Indeed, it is immediate from (1.8) and (1.1) that

$$
f(0)=f(1)=0 .
$$

Moreover,

$$
f^{\prime}(0)=-1+\sum_{k=2}^{\infty} k p_{k}=N-1 \quad \text { and } \quad f^{\prime}(1)=-1 .
$$

Finally, $f^{\prime \prime} \leq 0$ on $[0,1]$, so $\left.f\right|_{(0,1)}>0$ and $f(u) \leq f^{\prime}(0) u$ on $[0,1]$. Moreover, the moment bound (1.2) ensures that $f \in \mathcal{C}^{1, \beta}([0,1])$.

Let us mention that not every nonlinearity of the Fisher-KPP type comes from a branching process, and that convergence in (1.14) holds for all nonlinearities of the Fisher-KPP type, not just "probabilistic" ones. One reflection of the rigidity of the class of probabilistic nonlinearities is that they satisfy

$$
f^{\prime}(1)=-1
$$

and the Fisher-KPP property is not related to $f^{\prime}(1)$. Also, probabilistic nonlinearities are concave, which is not necessary for (1.13).

For later use, we introduce the decomposition

$$
f(u)=(N-1) u-F(u),
$$

so that $F$ denotes the "nonlinear" part of the reaction $f$. By $(1.16)$ and $(1.13), F^{\prime}(0)=0$ and $F(u)>0$ for all $u \in(0,1]$. When $f$ is probabilistic, $f^{\prime \prime} \leq 0$ and (1.17) imply that $F$ and $F^{\prime}$ are increasing and

$$
0=F^{\prime}(0)<F^{\prime}(u)<F^{\prime}(1)=N \quad \text { for all } u \in(0,1) .
$$

The connection between branching Brownian motion and the Fisher-KPP equation runs deeper than (1.5) and (1.6). Consider the measure-valued process that characterizes the BBM seen from the position $m(t)$ :

$$
\mathcal{X}_{t}=\sum_{k=1}^{n(t)} \delta_{m(t)-x_{k}(t)}
$$


recalling that $n(t)$ is the total number of particles alive at time $t$ and $x_{1}(t) \geq x_{2}(t) \geq \cdots \geq x_{n(t)}(t)$ are the positions of those particles. In the binary case $p_{2}=1,[1,3,7]$ show that

$$
\mathcal{X}_{t} \stackrel{\text { law }}{\longrightarrow} \mathcal{X}=\sum_{k \geq 1} \delta_{\chi_{k}} \quad \text { as } t \rightarrow \infty
$$

We use the convention $\chi_{1} \leq \chi_{2} \leq \ldots$ Due to the sign reversion in (1.20), $\chi_{1}$ corresponds to the rightmost particle in the original process, $\chi_{2}$ to the second largest, and so on. In the literature, $\mathcal{X}$ is often called the limiting extremal process (see [2], [3]).

In this paper, we examine the distance between the two leading particles $x_{1}(t)$ and $x_{2}(t)$. More precisely, we study the distance after the limit $t \rightarrow+\infty$ :

$$
d_{12}=\chi_{2}-\chi_{1} .
$$

The main result of this paper is the tail asymptotics of the random variable $d_{12}$.

Theorem 1.1 As $a \rightarrow \infty$, we have

$$
\mathbb{P}\left(d_{12}>a\right)=\frac{A \gamma_{*}}{2 \lambda_{*}^{2} \sqrt{\pi}}\left(\frac{a}{2 \sqrt{N}}\right)^{3 \sqrt{N} / 2} e^{-(\sqrt{N}+\sqrt{N-1})\left(a+\bar{x}_{0}\right)}\left[1+O\left(a^{-1 / 2}\right)\right]
$$

with $A$ as in (1.11).

To prove Theorem 1.1, we relate the distance $x_{1}(t)-x_{2}(t)$ to a derivative of the Fisher-KPP equation with respect to its initial data. The bulk of the proof is a detailed study of this derivative.

Weaker versions of the above result were already known. In [7], Derrida and the second author predicted the exponential order of (1.22) for binary BBM. Cortines, Hartung and Louidor confirmed this conjecture [8, Theorem 1.4], again in the binary case $N=2$ :

$$
\lim _{a \rightarrow \infty} a^{-1} \log \mathbb{P}\left(d_{12}>a\right)=-(\sqrt{2}+1) .
$$

(The limit given in [8] is in fact $-(\sqrt{2}+2)$, since the authors use Brownian motion of variance $t$ rather than 2t.) However, neither [7] nor [8] discuss the sub-exponential behavior of $\mathbb{P}\left(d_{12}>a\right)$. In particular, the algebraic pre-factor $a^{3 \sqrt{N} / 2}$ in Theorem 1.1 is new. We discuss the origin of this peculiar term in the next section.

More broadly, [7] is one of the main motivations for our study. It lays out numerous fascinating connections between the Bramson shifts of certain Fisher-KPP solutions and fine properties of branching Brownian motion. Some of these predictions have been addressed elsewhere [16], but many have not yet been proven rigorously.

We close with a word on notation: throughout the paper, we let $C>0$ and $c>0$ be constants which may change from line to line. We think of $C$ as large and $c$ as small.

\section{Acknowledgments}

CG was partially supported by the Fannie and John Hertz Foundation and NSF grant DGE-1656518, LM and LR by the US-Israel Binational Foundation, and LR by NSF grants DMS-1613603 and DMS1910023.

\section{Outline of the proof of Theorem 1.1}

We begin by relating the law of $d_{12}$ to the long-time behavior of a certain PDE solution. 


\section{An expression for the distribution of $d_{12}$}

Consider a branching Brownian motion shifted to start from a single particle at position $-a$ rather than 0 . Recall that $x_{1}(t) \geq x_{2}(t) \geq \cdots \geq x_{n(t)}(t)$ are the positions of the $n(t)$ particles alive at time $t$. We introduce the (unnormalized) density

$$
z(t, x ; a) \mathrm{d} x:=\mathbb{P}\left(x_{1}(t) \in[x, x+\mathrm{d} x], x_{2}(t)<x-a\right)
$$

under the convention that $x_{2}(t)=-\infty$ before the first branching event. We derive an evolution equation for $z$ by considering the dependence of $z(t+\varepsilon, x ; a)$ on the state of the system at the small time $\varepsilon>0$.

At time $\varepsilon$, there have been no branching events with probability $1-\varepsilon+o(\varepsilon)$, one branching event with probability $\varepsilon+o(\varepsilon)$, and more than one branching event with probability $o(\varepsilon)$. We will only track terms of order $\varepsilon$ or larger, so the last event can be safely discarded. In the first case, there is a single particle present at time $\varepsilon$. Its position is $-a+\eta \sqrt{\varepsilon}$, where $\eta$ is a centered Gaussian of variance 2. By the Markov property, the conditional probability that the event defining $z(t+\varepsilon, x ; a)$ is fulfilled is then $z(t, x-\eta \sqrt{\varepsilon} ; a)$.

In the second case, there are $k$ particles (with $k>1$ random) with positions $-a+O(\sqrt{\varepsilon})$ at time $\varepsilon$. Given this, we claim that the probability that the event defining $z(t+\varepsilon, x ; a)$ is fulfilled is

$$
k z(t, x ; a)[1-H(t, x)]^{k-1}+o(1) .
$$

Indeed, the descendant-BBM generated by one particle should satisfy the event defining

$$
z(t, x+O(\sqrt{\varepsilon}) ; a)=z(t, x ; a)+o(1),
$$

while the descendant-BBMs generated by the remaining $k-1$ particles should all lie to the left of $x-a$ at time $t+\varepsilon$. Each of these $k-1$ particles began near $-a$ at time $\varepsilon$, so the probability that its descendant-BBM lies to the left of $x-a$ at time $t+\varepsilon$ is $1-H(t, x)+o(1)$. Finally, the particles evolve independently after time $\varepsilon$ and there are $k$ combinatorial choices for the distinguished $z$-particle. The product formula (2.2) follows.

Gathering these events, we conclude that

$$
\begin{aligned}
z(t+\varepsilon, x ; a) & =\mathbb{E}\left[(1-\varepsilon) z(t, x-\eta \sqrt{\varepsilon} ; a)+\varepsilon k z(t, x ; a)[1-H(t, x)]^{k-1}+o(\varepsilon)\right] \\
& =(1-\varepsilon) z(t, x ; a)+\partial_{x}^{2} z(t, x ; a) \varepsilon+\varepsilon z(t, x ; a) \sum_{k \geq 2} p_{k} k[1-H(t, x)]^{k-1}+o(\varepsilon),
\end{aligned}
$$

where the expectation in the first line is taken over $\eta$ and $k$. Taking $\varepsilon \searrow 0$ and recalling the definition of $f$ in (1.8), we obtain

$$
\partial_{t} z(t, x ; a)=\partial_{x}^{2} z(t, x ; a)+f^{\prime}(H(t, x)) z(t, x ; a), \quad z(0, x ; a)=\delta(x+a) .
$$

By the definition of $z$,

$$
\mathbb{P}\left(x_{1}(t)-x_{2}(t)>a\right)=\int_{\mathbb{R}} z(t, x ; a) \mathrm{d} x
$$

Taking $t \rightarrow \infty$ :

$$
\mathbb{P}\left(d_{12}>a\right)=\lim _{t \rightarrow \infty} \int_{\mathbb{R}} z(t, x ; a) \mathrm{d} x .
$$

To understand the long-time behavior of $z$, we observe that (2.3) resembles a derivative of the Fisher-KPP equation (1.6). Indeed $\partial_{x} H$ solves (2.3) with different initial data. Bramson showed that 
$H$ converges to a traveling wave in the $m$-moving frame. If we apply standard parabolic estimates to $(1.9)$, we can conclude that

$$
\partial_{x} H(t, x+m(t)) \rightarrow U^{\prime}\left(x-\bar{x}_{0}\right)
$$

uniformly in $x \in \mathbb{R}$ as $t \rightarrow \infty$. This suggests that $U^{\prime}\left(x-\bar{x}_{0}\right)$ is a stable steady state at $t=\infty$ of (2.3) in the moving frame. Since (2.3) is linear, we might expect that $z$ converges to a multiple of this state in the moving frame. The methods of $[11,17]$ can be adapted to confirm these heuristics:

Proposition 2.1 For each $a>0$, there exists $M(a)>0$ such that

$$
z(t, x+m(t) ; a) \rightarrow-M(a) U^{\prime}\left(x-\bar{x}_{0}\right)
$$

uniformly in $x \in \mathbb{R}$ as $t \rightarrow \infty$.

This proposition is essentially a consequence of Proposition 4.1 and Lemma 5.1 in [17]; we omit the proof. Those results concern solutions to the Fisher-KPP equation, but (2.3) has a similar structure and the arguments adapt without difficulty.

To use (2.5) in (2.4), we need to commute the long-time limit and the spatial integral.

Lemma 2.2 For all $a>0$,

$$
\lim _{t \rightarrow \infty} \int_{\mathbb{R}} z(t, x+m(t) ; a) \mathrm{d} x=M(a) .
$$

Proof. Throughout, we fix $a>0$. By the comparison principle and (2.3), $z \geq 0$. Since (2.5) is uniform in $x$, it suffices to show that none of the mass of $z$ escapes to infinity. Recalling the definition (2.1), we have

$$
z(t, x ; a) \leq \mathbb{P}\left(x_{1}(t) \in[x, x+\mathrm{d} x]\right)=-\partial_{x} H(t, x+a) .
$$

Hence for $L>0,(1.9)$ and (1.11) imply that

$$
\int_{L}^{\infty} z(t, x+m(t) ; a) \mathrm{d} x \leq H(t, L+m(t)+a) \leq 2 U\left(L+a-\bar{x}_{0}\right) \leq C(a) e^{-c L},
$$

provided $t$ is sufficiently large (depending on $L$ and $a$ ). Similarly, (1.11) yields

$$
\int_{-\infty}^{-L} z(t, x+m(t) ; a) \mathrm{d} x \leq C(a) e^{-c L}
$$

when $t$ is large. Therefore (2.5) implies

$$
\begin{aligned}
\lim _{t \rightarrow \infty} \int_{\mathbb{R}} z(t, x+m(t) ; a) \mathrm{d} x & =\lim _{t \rightarrow \infty} \int_{-L}^{L} z(t, x+m(t) ; a) \mathrm{d} x+O\left(e^{-c L}\right) \\
& =M(a)\left[U\left(-L-\bar{x}_{0}\right)-U\left(L-\bar{x}_{0}\right)\right]+O\left(e^{-c L}\right) \\
& =M(a)+O\left(e^{-c L}\right),
\end{aligned}
$$

where we have again used (1.11). Since $L>0$ was arbitrary, (2.6) follows.

Combining (2.4) and (2.6), we obtain $\mathbb{P}\left(d_{12}>a\right)=M(a)$. Hence Proposition 2.1 becomes

$$
z(t, x+m(t) ; a) \rightarrow-\mathbb{P}\left(d_{12}>a\right) U^{\prime}\left(x-\bar{x}_{0}\right) \quad \text { as } t \rightarrow \infty
$$

uniformly in $x \in \mathbb{R}$. We will use this long-time limit to determine the the dependence of $\mathbb{P}\left(d_{12}>a\right)$ on $a$. First, however, we describe a connection with the Bramson shift introduced in (1.14). 


\section{The distribution of $d_{12}$ through Bramson shifts}

As noted above, (2.3) appears to be a derivative of (1.6). Precisely, it is the derivative with respect to a certain perturbation of the initial data $\mathbb{1}_{(-\infty, 0]}$. Consider the initial condition

$$
u(0, x ; y, a)=\mathbb{1}_{(-\infty, 0]}(x)-\mathbb{1}_{(y-a,-a]}(x)
$$

parameterized by $y<0$ and $a>0$. We emphasize that $y$ is negative, so $y-a<-a$. We think of $a$ as fixed, but allow $y$ to vary. Note that

$$
u(0, x ; 0, a)=\mathbb{1}_{(-\infty, 0]}(x)
$$

for all $a>0$ and

$$
\partial_{y} u(0, x ; y, a)=\delta(x-y+a)
$$

in the distributional sense. Now let $u(t, x ; y, a)$ denote the solution to the Fisher-KPP equation (1.12) with initial data (2.8). In particular, (1.7) and (2.9) imply that

$$
u(t, x ; 0, a)=H(t, x)
$$

for all $a>0$.

Now, standard parabolic estimates imply that $u$ is differentiable in $y$ and that its derivative satisfies the appropriate derivative of (1.12). That is, if

$$
z(t, x ; y, a)=\partial_{y} u(t, x ; y, a)
$$

then (2.10) yields

$$
\partial_{t} z(t, x ; y, a)=\partial_{x}^{2} z(t, x ; y, a)+f^{\prime}(u(t, x ; y, a)) z(t, x ; y, a), \quad z(0, x ; y, a)=\delta(x-y+a) .
$$

In particular, $(2.11)$ and uniqueness imply that $z(t, x ; 0, a)=z(t, x ; a)$. That is, the distribution of $x_{1}(t)-x_{2}(t)$ can be expressed via derivatives of the Fisher-KPP equation with respect to its initial data. This connection allows us to relate the law of $d_{12}$ to the Bramson shift in (1.14).

Recall that for each fixed $y$ and $a,(1.14)$ implies that

$$
u(t, x+m(t) ; y, a) \rightarrow U(x-s(y, a))
$$

uniformly in $x$ as $t \rightarrow \infty$, where $s(y, a)=s[u(0, x ; y, a)]$ is the Bramson shift associated with the initial condition $u(0, x ; y, a)$. This is well defined because $u(0, x ; y, a)$ is bounded, nonnegative, and compactly supported on the right. In Appendix B, we show that $s$ is differentiable in $y$ and that $\partial_{y}$ commutes with the long-time limit (2.13). Hence (2.12) and (2.13) imply

$$
\begin{aligned}
\lim _{t \rightarrow \infty} z(t, x+m(t) ; a) & =\left.\lim _{t \rightarrow \infty} \partial_{y} u(t, x+m(t) ; y, a)\right|_{y=0} \\
& =\left.\partial_{y}\left(\lim _{t \rightarrow \infty} u(t, x+m(t) ; y, a)\right)\right|_{y=0} \\
& =\left.\partial_{y} U(x-s(y, a))\right|_{y=0}=-\partial_{y} s(0, a) U^{\prime}\left(x-\bar{x}_{0}\right)
\end{aligned}
$$

In the last line, we used the identity $s(0, a)=\bar{x}_{0}$, which follows from (1.15) and (2.9). Comparing with (2.7), we see that

$$
\mathbb{P}\left(d_{12}>a\right)=\partial_{y} s(0, a) .
$$

That is, the gap between the two leaders in the BBM extremal process is encoded in the Bramson shifts $s(y, a)$. 
This can be seen directly from ideas of [7] and [16]. Using (2.8) and McKean's identity [15], we have

$$
\begin{aligned}
1-u(t, x+m(t) ; y, a) & =\mathbb{E}\left[\prod_{k=1}^{n(t)}\left(1-u\left(0, x+m(t)-x_{k}(t) ; y, a\right)\right)\right] \\
& =\mathbb{P}\left[x+m(t)-x_{k}(t) \in(y-a,-a] \cup(0, \infty) \quad \text { for all } 1 \leq k \leq n(t)\right] \\
& =\mathbb{P}\left[m(t)-x_{k}(t) \in(y-a-x,-a-x] \cup(-x, \infty) \quad \text { for all } 1 \leq k \leq n(t)\right] .
\end{aligned}
$$

Take the limit $t \rightarrow \infty,(2.13)$ and (1.21) imply

$$
\begin{aligned}
1-U(x-s(y, a)) & =\mathbb{P}\left[\chi_{k} \in(y-a-x,-a-x] \cup(-x, \infty) \quad \text { for all } k \geq 1\right] \\
& =\mathbb{P}\left[\chi_{1}>-x\right]+\mathbb{P}\left[\chi_{1} \in(y-a-x,-a-x], \chi_{2}>-x\right]+O\left(y^{2}\right),
\end{aligned}
$$

where we used $\chi_{1} \leq \chi_{2} \leq \ldots$. The $O\left(y^{2}\right)$ term contains all events with multiple particles in $(y-a-x,-a-x]$. The first term in the right side of $(2.16)$ is $1-U\left(x-\bar{x}_{0}\right)=1-U(x-s(0, a))$. Moving this term to the left side and dividing by $|y|$, we obtain in the $y \nearrow 0$ limit

$$
\begin{aligned}
\left.\partial_{y} U(x-s(y, a))\right|_{y=0}=-\partial_{y} s(0, a) U^{\prime}\left(x-\bar{x}_{0}\right) & =\lim _{y \nearrow 0} \frac{\mathbb{P}\left[\chi_{1} \in(y-a-x,-a-x], \chi_{2}>-x\right]}{|y|} \\
& =\frac{1}{\mathrm{~d} x} \mathbb{P}\left[-\left(\chi_{1}+a\right) \in[x, x+\mathrm{d} x), d_{12}>a\right] .
\end{aligned}
$$

Integrating over $x$, we recover (2.15). Thus, our work adds to a growing body of literature identifying properties of the extremal process with long-time behavior of the Fisher-KPP equation. For results of a similar flavor, see [7] and [16].

\section{Long-time asymptotics for $z$}

The previous subsection puts our problem in a broader context, but it is not essential to our proof of Theorem 1.1. Instead, we can proceed directly from (2.7), which states that $\mathbb{P}\left(d_{12}>a\right)$ is the coefficient of $-U^{\prime}$ in the long-time limit of $z$ in the $m$-moving frame. We must determine the dependence of this coefficient on $a$ as $a \rightarrow \infty$. To do so, it helps to shift by $m$ and remove the exponential decay of $U^{\prime}$. We define

$$
r(t, x)=(t+1)^{-3 / 2} e^{\lambda_{*}(x+a)} z(t, m(t)+x ; a) .
$$

The factor of $(t+1)^{-3 / 2}$ above is a matter of convenience which will be explained below. Of course, $r$ depends on $a$ as well, but explicit dependence will become cumbersome in the remainder of the paper, so we drop it.

Using (1.3) and (1.4), we see that the function $r(t, x)$ satisfies

$$
\partial_{t} r=\partial_{x}^{2} r-\frac{3}{2 \lambda_{*}(t+1)} \partial_{x} r-\left[N-1-f^{\prime}(H(t, m(t)+x))\right] r, \quad r(0, x)=\delta(x+a) .
$$

Recalling the decomposition (1.18) of $f$, we see that the coefficient of the reaction term is $-F^{\prime}(H)$. For concision, we introduce the notation

$$
V(t, x)=F^{\prime}(H(t, m(t)+x))
$$


and write $(2.18)$ as

$$
\partial_{t} r=\partial_{x}^{2} r-\frac{3}{2 \lambda_{*}(t+1)} \partial_{x} r-V(t, x) r, \quad r(0, x)=\delta(x+a) .
$$

By (1.19), $0<V<N$ and

$$
\begin{array}{ll}
V(t, x) \rightarrow N & \text { as } x \rightarrow-\infty, \\
V(t, x) \rightarrow 0 & \text { as } x \rightarrow+\infty .
\end{array}
$$

Moreover,

$$
V(t, x) \rightarrow V(\infty, x)=F^{\prime}\left(U\left(x-\bar{x}_{0}\right)\right) \quad \text { as } t \rightarrow \infty .
$$

The potential $V$ largely confines $r$ to $\mathbb{R}_{+}$. Since the drift term in (2.20) decays in time, we can thus view (2.20) as a heat equation on $\mathbb{R}_{+}$with (approximate) Dirichlet conditions at $x=0$. It follows that the dynamics of $r$ are largely driven at the diffusive scale $x \asymp \sqrt{t}$, by which we mean $c \sqrt{t} \leq x \leq C \sqrt{t}$. In particular, at that scale we should expect $r$ to resemble a multiple of the fundamental solution to the Dirichlet heat equation on $\mathbb{R}_{+}$:

$$
r(t, x) \sim \widetilde{M}(a) \frac{x}{(t+1)^{3 / 2}} e^{-x^{2} / 4(t+1)} \quad \text { for } t \gg 1, x \asymp \sqrt{t}
$$

and some $\widetilde{M}(a)>0$.

On the other hand, the asymptotics (1.11) for $U$ extend naturally to $U^{\prime}$. In particular,

$$
U^{\prime}\left(x-\bar{x}_{0}\right) \sim-\lambda_{*} x e^{-\lambda_{*}\left(x-\bar{x}_{0}\right)} \quad \text { as } x \rightarrow \infty .
$$

Thus if $x$ is large but fixed, (2.5), (2.17), and (2.23) suggest that

$$
r(t, x) \approx \lambda_{*} e^{\lambda_{*}\left(\bar{x}_{0}+a\right)} \mathbb{P}\left(d_{12}>a\right) \frac{x}{(t+1)^{3 / 2}} \quad \text { as } t \rightarrow \infty .
$$

Comparing (2.22) and (2.24), we expect

$$
\widetilde{M}(a)=\lambda_{*} e^{\lambda_{*}\left(\bar{x}_{0}+a\right)} \mathbb{P}\left(d_{12}>a\right) .
$$

Once again, the methods of $[11,17]$ confirm these calculations:

Proposition 2.3 For each $a>0$ and $\gamma \in(0,1 / 2)$, there exist $C(a, \gamma)>0$ and $h:[0, \infty) \rightarrow \mathbb{R}$ such that for all $t \geq 0$ and $x \in \mathbb{R}$,

$$
r(t, x)=\left[\lambda_{*} e^{\lambda_{*}\left(\bar{x}_{0}+a\right)} \mathbb{P}\left(d_{12}>a\right)+h(t)\right] \frac{x_{+}}{(t+1)^{3 / 2}} e^{-x^{2} / 4(t+1)}+R(t, x)
$$

with

$$
|R(t, x)| \leq C(a, \gamma)(t+1)^{-(3 / 2-\gamma)} e^{-x^{2} /[6(t+1)]} \quad \text { and } \quad h(t) \rightarrow 0 \quad \text { as } t \rightarrow \infty .
$$

We have used the notation $x_{+}=\max \{x, 0\}$ in (2.25). Again, this proposition is very similar to Proposition 4.1 and Lemma 5.1 in [17], so we omit the proof. 


\section{A moment computation}

By (2.15) and (2.25), it now suffices to understand the long-time dynamics of $r$ on the diffusive scale. We capture these dynamics in a certain moment of $r$. An elementary computation using (2.25) and (2.26) shows that

$$
\mathbb{P}\left(d_{12}>a\right)=\frac{1}{2 \lambda_{*} \sqrt{\pi}} e^{-\lambda_{*}\left(a+\bar{x}_{0}\right)} \lim _{t \rightarrow \infty} \int_{0}^{\infty} x r(t, x) \mathrm{d} x .
$$

This identity is the reason for introducing the $(t+1)^{-3 / 2}$ factor in (2.17). It is convenient to express this moment in terms of the function

$$
\psi(x)=-U_{0}^{\prime}(x) e^{\lambda_{*} x}
$$

where we have introduced the notation

$$
U_{0}(x)=U\left(x-\bar{x}_{0}\right)
$$

for the limiting shift of the front $U$. The function $\psi$ is a positive time-independent solution to the leading-order part of (2.20):

$$
0=\psi^{\prime \prime}-V(\infty, x) \psi=\psi^{\prime \prime}-F^{\prime}\left(U_{0}(x)\right) \psi
$$

Here we have used (2.21) and (2.28) to express the long-time limit of the potential $V$ introduced in (2.19). Recalling that $\gamma_{*}+\lambda_{*}=\sqrt{N},(1.11)$ and an elementary ODE argument imply that

$$
\begin{array}{ll}
\psi(x)=A \gamma_{*} e^{-\gamma_{*} \bar{x}_{0}} e^{\sqrt{N} x}+O\left(e^{(\sqrt{N}+c) x}\right) & \text { as } x \rightarrow-\infty \\
\psi(x)=\lambda_{*} e^{\lambda_{*} \bar{x}_{0}} x+O\left(e^{-c x}\right) & \text { as } x \rightarrow+\infty
\end{array}
$$

for some $c>0$. It follows from (2.25), (2.26), and (2.30) that (2.27) can be written as

$$
\mathbb{P}\left(d_{12}>a\right)=\frac{1}{2 \lambda_{*}^{2} \sqrt{\pi}} e^{-\lambda_{*}\left(a+2 \bar{x}_{0}\right)} \lim _{t \rightarrow+\infty} I(t),
$$

where

$$
I(t)=\int_{\mathbb{R}} \psi(x) r(t, x) \mathrm{d} x .
$$

This completes our series of reductions: to control $\mathbb{P}\left(d_{12}>a\right)$, we determine the dependence of $I(\infty)$ on $a$.

Motivated by (2.21), we write (2.20) as

$$
\partial_{t} r=\partial_{x}^{2} r-\frac{3}{2 \lambda_{*}(t+1)} \partial_{x} r-V(\infty, x) r+E(t, x) r, \quad r(0, x)=\delta(x+a),
$$

with an error term

$$
E(t, x)=V(\infty, x)-V(t, x) .
$$

If we multiply (2.33) by $\psi$ and integrate, (2.29) yields

$$
\frac{\mathrm{d} I}{\mathrm{~d} t}(t)=\frac{3}{2 \lambda_{*}(t+1)} \int_{\mathbb{R}} r(t, x) \psi^{\prime}(x) \mathrm{d} x+\int_{\mathbb{R}} E(t, x) r(t, x) \psi(x) \mathrm{d} x .
$$

We will see below that the second term in the right side of (2.35) is, indeed, an error term, so we focus on the first term. To this end, we describe the dynamics of (2.33) qualitatively, ignoring 
the error term $E r$. First note that the mass of the solution on $\mathbb{R}_{-}$will decay exponentially in time under (2.33), due to absorption from the term $-F^{\prime}\left(U_{0}\right) r$. After all, (1.19) implies $F^{\prime}\left(U_{0}\right)>0$ and, in particular, $F^{\prime}(1)=N$. However, mass that escapes to $\mathbb{R}_{+}$experiences almost no absorption because $F^{\prime}(0)=0$. This "fugitive" mass diffuses, but gets absorbed whenever it returns to $\mathbb{R}_{-}$. Thus, as noted previously, (2.33) acts much like the heat equation on $\mathbb{R}_{+}$with a Dirichlet boundary condition at $x=0$. That said, there is initially no mass on $\mathbb{R}_{+}$, so we must include an initial time layer during which the mass escapes from $\mathbb{R}_{-}$to $\mathbb{R}_{+}$.

The initial condition $r(0, x)=\delta(x+a)$ is "deep in the large absorption territory," and takes a while to diffuse to $\mathbb{R}_{+}$. If we neglect the drift in the right side of $(2.33)$, then, in the absence of absorption, the proportion of mass that diffuses from position $-a$ to $\mathbb{R}_{+}$at time $t$ is roughly $e^{-a^{2} /(4 t)}$. Attrition by the absorbing potential approximately reduces this by the factor $e^{-N t}$. Thus to leading order, the mass that escapes to $\mathbb{R}_{+}$at time $t$ is

$$
\exp \left(-N t-a^{2} /(4 t)\right)
$$

Therefore, the mass flux into $\mathbb{R}_{+}$is maximized at the time

$$
t_{*}=\frac{a}{2 \sqrt{N}}
$$

and this is, roughly, the size of the initial time layer.

Let us now use this heuristic to approximate the first term in the right side of (2.35):

$$
\frac{3}{2 \lambda_{*}(t+1)} \int_{\mathbb{R}} r(t, x) \psi^{\prime}(x) \mathrm{d} x=\frac{3}{2 \lambda_{*}(t+1)} \int_{\mathbb{R}} \frac{\psi^{\prime}(x)}{\psi(x)} \psi(x) r(t, x) \mathrm{d} x .
$$

We see from (2.30) that

$$
\frac{\psi^{\prime}(x)}{\psi(x)} \sim \begin{cases}\sqrt{N} & \text { for } x \ll-1 \\ \frac{1}{x} & \text { for } x \gg 1\end{cases}
$$

The heuristic argument above suggests that most of the mass lies in $\mathbb{R}_{-}$until $t_{*}$, when it transfers to $\mathbb{R}_{+}$. Therefore, we expect that

$$
\int_{\mathbb{R}} \frac{\psi^{\prime}(x)}{\psi(x)} \psi(x) r(t, x) \mathrm{d} x \approx \sqrt{N} \int_{\mathbb{R}} \psi(x) r(t, x) \mathrm{d} x \quad \text { for } t<t_{*} .
$$

After $t_{*}$, the mass of $r$ will largely stay in $\mathbb{R}_{+}$, and will spread to the diffusive scale $x \asymp \sqrt{t}$. Since $\frac{\psi^{\prime}}{\psi} \approx \frac{1}{x}$, we should have

$$
\int_{\mathbb{R}} \frac{\psi^{\prime}(x)}{\psi(x)} \psi(x) r(t, x) \mathrm{d} x \ll \int_{\mathbb{R}} \psi(x) r(t, x) \mathrm{d} x \quad \text { for } t>t_{*} .
$$

Thus, $I(t)$ satisfies

$$
\frac{\mathrm{d} I}{\mathrm{~d} t}(t) \approx \frac{3 \sqrt{N}}{2 \lambda_{*}(t+1)} I(t) \quad \text { for } t<t_{*}, \quad \frac{\mathrm{d} I}{\mathrm{~d} t}(t) \approx 0 \quad \text { for } t>t_{*} .
$$

Integrating, we find

$$
\lim _{t \rightarrow+\infty} I(t) \approx t_{*}^{3 \sqrt{N} /\left(2 \lambda_{*}\right)} I(0)=\left(\frac{a}{2 \sqrt{N}}\right)^{3 \sqrt{N} /\left(2 \lambda_{*}\right)} I(0)
$$

when $a \gg 1$. In the remainder of this paper, we justify (2.37). 
Proposition 2.4 We have

$$
\lim _{t \rightarrow+\infty} I(t)=\left(\frac{a}{2 \sqrt{N}}\right)^{3 \sqrt{N} /\left(2 \lambda_{*}\right)}\left[1+O\left(a^{-1 / 2}\right)\right] \int_{\mathbb{R}} \psi(x) r(0, x) \mathrm{d} x \quad \text { as } a \rightarrow+\infty .
$$

Theorem 1.1 is a consequence of Proposition 2.4. Indeed, using (2.30), we see that the integral in the right side of $(2.38)$ is

$$
\int_{\mathbb{R}} \psi(x) r(0, x) \mathrm{d} x=\psi(-a)=A \gamma_{*} e^{-\gamma_{*} \bar{x}_{0}} e^{-\sqrt{N} a}\left(1+o\left(e^{-c a}\right)\right)
$$

for some $c>0$. We then use (2.31) to write

$$
\begin{aligned}
\mathbb{P}\left(d_{12}>a\right) & =\frac{1}{2 \lambda_{*}^{2} \sqrt{\pi}} e^{-\lambda_{*}\left(a+2 \bar{x}_{0}\right)} \lim _{t \rightarrow+\infty} I(t) \\
& =\frac{A \gamma_{*}}{2 \lambda_{*}^{2} \sqrt{\pi}} e^{-\left(2 \lambda_{*}+\gamma_{*}\right) \bar{x}_{0}}\left(\frac{a}{2 \sqrt{N}}\right)^{3 \sqrt{N} /\left(2 \lambda_{*}\right)} e^{-\left(\lambda_{*}+\sqrt{N}\right) a}\left[1+O\left(a^{-1 / 2}\right)\right]\left(1+o\left(e^{-c a}\right)\right) \\
& =\frac{A \gamma_{*}}{2 \lambda_{*}^{2} \sqrt{\pi}}\left(\frac{a}{2 \sqrt{N}}\right)^{3 \sqrt{N} /\left(2 \lambda_{*}\right)} e^{-(\sqrt{N}+\sqrt{N-1})\left(a+\bar{x}_{0}\right)}\left[1+O\left(a^{-1 / 2}\right)\right] .
\end{aligned}
$$

This finishes the proof of Theorem 1.1.

The rest of the paper contains the proof of Proposition 2.4. The strategy is to estimate the ratio $\dot{I}(t) / I(t)$ on various times scales. Lemmas 4.1, 5.1, and 6.1 below express these estimates. At the end of Section 6, we collect these results and prove Proposition 2.4. We note that Proposition 2.4 likely holds for general Fisher-KPP nonlinearities, but the significance of the left side is far from clear when $f$ is not probabilistic. In our proof, we confine our attention to probabilistic $f$. Finally, because we are interested in the regime $a \rightarrow \infty$, we always implicitly assume that $a \geq 1$.

\section{The time scales and the correctors}

We now turn to the proof of Proposition 2.4. In this section, we discuss the time scales on which various approximations to the dynamics of (2.20) should be valid, and introduce the corresponding "scattering decomposition" of the solution.

\section{The time scales}

Let us first explain the time scales on which various effects will dominate. Our previous reasoning indicates that at times $t<t_{*}$, the heat equation has not had enough time to diffuse much mass from the initial position $x=-a$ to $x \geq 0$. The evolution of $r(t, x)$, the solution to $(2.20)$, is thus dominated by its homogeneous part

$$
\partial_{t} p=\partial_{x}^{2} p-\frac{3}{2 \lambda_{*}(t+1)} \partial_{x} p-N p, \quad p(0, x)=\delta(x+a),
$$

as $F^{\prime}(1)=N$. Its explicit solution is

$$
p(t, x)=\frac{1}{\sqrt{4 \pi t}} \exp \left\{-N t-\frac{1}{4 t}\left[x+a-\frac{3}{2 \lambda_{*}} \log (t+1)\right]^{2}\right\} .
$$

The corrector

$$
q(t, x)=r(t, x)-p(t, x)
$$


solves

$$
\partial_{t} q=\partial_{x}^{2} q-\frac{3}{2 \lambda_{*}(t+1)} \partial_{x} q-V(t, x) q+(N-V(t, x)) p, \quad q(0, x)=0,
$$

recalling that

$$
V(t, x)=F^{\prime}(H(t, x+m(t)) .
$$

Since $h^{\prime}(u) \leq N$ by $(1.19), V \leq N$. It follows that

$$
q(t, x) \geq 0
$$

We view (2.20) as a perturbation of the absorbing heat equation (3.1), so that $p(t, x)$ represents the free evolution and $q(t, x)$ accounts for the interaction with the potential $V(t, x)$. The role of $p$ is to transport the mass from $x=-a$ to the half-line $\mathbb{R}_{+}$, and the role of $q$ is to account for this escaped mass as $t \rightarrow \infty$. Indeed, we will show that

$$
I(t)=\int_{\mathbb{R}} r(t, x) \psi(x) \mathrm{d} x \approx \int_{\mathbb{R}} p(t, x) \psi(x) \mathrm{d} x \quad \text { for } t \ll t_{*}
$$

and

$$
I(t)=\int_{\mathbb{R}} r(t, x) \psi(x) \mathrm{d} x \approx \int_{\mathbb{R}} q(t, x) \psi(x) \mathrm{d} x \quad \text { for } t \gg t_{*} .
$$

In constructing our time scales, we must also consider the forcing term $(N-V) p$ in $(3.3)$. In particular, we study its moment contribution

$$
\int_{\mathbb{R}}(N-V(t, x)) \psi(x) p(t, x) \mathrm{d} x .
$$

To understand the time scales on which it may potentially play a role, note that the first two terms in the integrand decay on the left: $\psi(x)$ has the asymptotic behavior as in (2.30), and the first factor is controlled by the following lemma.

Lemma 3.1 There exist $B>0$ and $c>0$ depending only on $f$ such that

$$
0 \leq N-V(t, x) \leq \min \left\{B e^{\gamma_{*} x}, N\right\} \quad \text { and } \quad 0 \leq V(t, x) \leq \min \left\{B e^{-c x}, N\right\}
$$

for all $t \geq 0$ and $x \in \mathbb{R}$.

Proof. For $t>0$, define the shift $\tilde{m}(t)$ by

$$
H(t, \tilde{m}(t))=U_{0}(0) .
$$

This is well-defined and continuous because $H(t, \cdot)$ strictly decreases from 1 to 0 and $H$ is continuous when $t>0$. By Theorem 12 in [12],

$$
U_{0}(x) \leq H(t, x+\tilde{m}(t)) \leq 1 \text { for all } t>0 \text { and } x \leq 0
$$

and

$$
0 \leq H(t, x+\tilde{m}(t)) \leq U_{0}(x) \text { for all } t>0 \text { and } x \geq 0 .
$$

Bramson's work $[4,5]$ implies that $\tilde{m}(t)-m(t) \rightarrow 0$ as $t \rightarrow \infty$. Using (1.11) and (3.7), we find

$$
0 \leq 1-H(t, x+m(t)) \leq \min \left\{C e^{\gamma_{*} x}, 1\right\} \quad \text { for all } t \geq 0 \text { and } x \in \mathbb{R}
$$


for some $C$ depending only on $f$. Similarly, (1.11) and (3.8) imply

$$
0 \leq H(t, x+m(t)) \leq \min \left\{C e^{-\lambda_{*} x / 2}, 1\right\} \quad \text { for all } t \geq 0 \text { and } x \in \mathbb{R} .
$$

Now, (1.8) and (1.2) imply that $f$ is $\mathcal{C}^{2}$ away from 0 and $\mathcal{C}^{1, \beta}$ near 0 . The decomposition (1.18) implies the same regularity for $F$. Using the definition (2.19) of $V$, the first bound in (3.6) follows from (3.9), (1.19), and $F^{\prime} \in \mathcal{C}^{1}([1 / 2,1])$. The second bound in (3.6) follows from (3.10), (1.19), and $F^{\prime} \in \mathcal{C}^{\beta}([0,1])$.

To understand the spatial decay of $p(t, x)$, it helps to write it as

$$
p(t, x)=\Lambda(t ; a) e^{-a x /(2 t)} g(t, x)
$$

with the factor

$$
\Lambda(t ; a)=\frac{(t+1)^{3 a /\left(4 \lambda_{*} t\right)}}{\sqrt{4 \pi t}} e^{-N t-a^{2} /(4 t)}
$$

and the re-centered Gaussian

$$
g(t, x)=\exp \left\{-\frac{1}{4 t}\left[x-\frac{3}{2 \lambda_{*}} \log (t+1)\right]^{2}\right\}
$$

Combining (2.30), (3.6), and (3.11), we see that it is straightforward to control the spatial decay of the integrand in (3.5) as $x \rightarrow-\infty$ for times $t$ such that

$$
\gamma_{*}+\sqrt{N}>\frac{a}{2 t}
$$

i.e.

$$
t>\frac{a}{2(2 \sqrt{N}-\sqrt{N-1})} .
$$

Accordingly, we fix

$$
\xi_{-} \in\left(\frac{1}{2(2 \sqrt{N}-\sqrt{N-1})}, \frac{1}{2 \sqrt{N}}\right), \quad t_{-}=\xi_{-} a .
$$

Let us decompose the solution to (3.3) as

$$
q=q_{\mathrm{e}}+q_{\mathrm{m}},
$$

where $q_{\mathrm{e}}$ is forced on the time interval $\left[0, t_{-}\right]$and $q_{\mathrm{m}}$ on $\left[t_{-}, \infty\right)$ :

$$
\partial_{t} q_{\mathrm{e}}=\partial_{x}^{2} q_{\mathrm{e}}-\frac{3}{2 \lambda_{*}(t+1)} \partial_{x} q_{\mathrm{e}}-V(t, x) q_{\mathrm{e}}+(N-V(t, x)) \mathbb{1}_{\left[0, t_{-}\right]}(t) p
$$

and

$$
\partial_{t} q_{\mathrm{m}}=\partial_{x}^{2} q_{\mathrm{m}}-\frac{3}{2 \lambda_{*}(t+1)} \partial_{x} q_{\mathrm{m}}-V(t, x) q_{\mathrm{m}}+(N-V(t, x)) \mathbb{1}_{\left[t_{-}, \infty\right)}(t) p,
$$

with $q_{\mathrm{e}}(0, \cdot)=q_{\mathrm{m}}(0, \cdot)=0$.

As we have discussed, the product $(N-V) p$ is very small for $t \ll t_{*}$, and $t_{-} \ll t_{*}$ if $a \gg 1$. It follows that $q_{\mathrm{e}}$ should never form a significant part of $r$, and we think of it as error. In contrast, $q_{\mathrm{m}}$ is eventually the principal part of $r$, so we view it as the main part of $q$.

Although $q_{\mathrm{e}}$ should be irrelevant, it is challenging to estimate. We introduced the corrector $q$ because it is easier to analyze the long-time behavior of adjoint-weighted mass which begins in $\mathbb{R}_{+}$, rather than deep in $\mathbb{R}_{-}$. As we argue above, the adjoint-weighted forcing $(N-V) \psi p$ for $q_{\mathrm{m}}$ is 
concentrated on $\mathbb{R}_{+}$. However, this is not the case for $q_{\mathrm{e}}$, which is still primarily forced deep in $\mathbb{R}_{-}$. We appear to be no better off than when we started with a point mass at $-a$ ! And indeed, we will be forced to control $q_{\mathrm{e}}$ using a further corrector.

However, $q_{\mathrm{e}}$ is driven by the forcing $(N-V) p$, which is far smaller than $p$ due to Lemma 3.1. It follows that $q_{\mathrm{e}}$ is much smaller than the original solution $r$. We can therefore be less precise in our estimation of $q_{\mathrm{e}}$. This wiggle room saves us from a futile infinite descent of correctors. Instead, two steps suffice. The details are rather technical, so we defer them to Section 7. There, we show:

Lemma 3.2 There exist $C, c>0$ independent of $a$ and $t$ such that for all $a \geq 1$ and $t \geq 0$ :

$$
\begin{aligned}
& \int_{\mathbb{R}} \psi(x) q_{\mathrm{e}}(t, x) \mathrm{d} x \leq C e^{-(\sqrt{N}+c) a}, \\
& \int_{\mathbb{R}} \psi^{\prime}(x) q_{\mathrm{e}}(t, x) \mathrm{d} x \leq \frac{C}{\sqrt{t+1}} e^{-(\sqrt{N}+c) a}, \\
& \int_{\mathbb{R}} \psi(x)|E(t, x)| q_{\mathrm{e}}(t, x) \mathrm{d} x \leq \frac{C}{(t+1)^{2}} e^{-(\sqrt{N}+c) a} .
\end{aligned}
$$

(We note that $\psi$ and $\psi^{\prime}$ are non-negative, so the first two bounds are effective.) These bounds feature an extra factor of $e^{-c a}$ relative to the main term, which is of order $e^{-\sqrt{N} a} ; C f$. (2.39). This justifies our treatment of $q_{\mathrm{e}}$ as error.

To prove Lemma 3.2, we will make use of the following result, which will also be useful in subsequent sections:

Lemma 3.3 Let $w(t, x)$ satisfy

$$
\begin{aligned}
& \partial_{t} w \leq \partial_{x}^{2} w-\frac{3}{2 \lambda_{*}(t+1)} \partial_{x} w-\alpha^{2} \mathbb{1}_{(-\infty,-K)}(x) w, \quad t>s, x \in \mathbb{R}, \\
& w(s, x) \leq \begin{cases}e^{-\kappa_{-} x} & \text { for } x<0, \\
e^{-\kappa_{+} x} e^{-x^{2} /(8 s)} & \text { for } x \geq 0,\end{cases}
\end{aligned}
$$

for some $\alpha>0, K \geq 0, \kappa_{-}<\alpha$, and $\kappa_{+}>0$. Then there exists a constant $C>0$ which depends on $\alpha, K$, and $\kappa_{-}$but not on $s$ or $\kappa_{+}$such that for all $t \geq s$, we have

$$
\begin{aligned}
& \int_{\mathbb{R}} \psi(x) w(t, x) \mathrm{d} x \leq C \max \left\{\kappa_{+}^{-2}, 1\right\}, \\
& \int_{\mathbb{R}} \psi^{\prime}(x) w(t, x) \mathrm{d} x \leq C \max \left\{\kappa_{+}^{-2}, 1\right\}(t-s+1)^{-1 / 2}, \\
& \int_{\mathbb{R}}|E(t, x)| \psi(x) w(t, x) \mathrm{d} x \leq C \max \left\{\kappa_{+}^{-2}, 1\right\}(t+1)^{-1 / 2}(t-s+1)^{-3 / 2} .
\end{aligned}
$$

We prove Lemma 3.3 in Appendix A, but offer a heuristic explanation here. Roughly speaking, we may think of (3.16) as the heat equation on the half-line with Dirichlet boundary conditions, so that

$$
w(t, x) \sim \frac{x}{(t-s+1)^{3 / 2}} e^{-x^{2} /(4(t-s+1))} \int_{0}^{\infty} x w(s, x) \mathrm{d} x \leq \frac{C}{\kappa_{+}^{2}} \frac{x}{(t-s+1)^{3 / 2}} e^{-x^{2} /(4(t-s+1))} .
$$

The bounds in (3.18)-(3.20) come from the right side of (3.21).

Going forward, we separately consider three regimes delimited by $t_{*}$ from (2.36) and $a$ : the early times $0 \leq t \leq t_{*}$ in Section 4, the middle times $t_{*} \leq t \leq a$ in Section 5 , and the late times $t \geq a$ in Section 6 . The cutoff $a$ is more or less arbitrary: it simply allows us to assume that $t=O(a)$ in the middle regime. As stated above, Proposition 2.4 and Theorem 1.1 are immediate consequences of Lemmas 4.1, 5.1, and 6.1. 


\section{The early times}

In this section, we start analyzing the contributions of $p$ and $q=q_{\mathrm{m}}+q_{\mathrm{e}}$ to $I(t)$ and $\dot{I}(t)$. We begin with the early times $t \leq t_{*}$. The terms involving the "early" corrector $q_{\mathrm{e}}$ have already been bounded in Lemma 3.2 and will turn out to be irrelevant - they are much smaller than the corresponding contributions of $p(t, x)$ in Lemma 4.2 below. It remains to estimate the terms involving $p(t, x)$ and $q_{\mathrm{m}}(t, x)$. We will see that the terms involving $p$ dominate for nearly the entire interval $t \in\left[0, t_{*}\right]$ (in particular, $q_{\mathrm{m}} \equiv 0$ for $\left.t \leq t_{-}\right)$. However, the contributions of $p$ and $q_{\mathrm{m}}$ become comparable when $t_{*}-t=O(\sqrt{a})$.

The main result of this section is the following lemma.

Lemma 4.1 We have

$$
\frac{\dot{I}(t)}{I(t)}=\frac{3 \sqrt{N}}{2 \lambda_{*}(t+1)}\left[1+O\left(e^{-\frac{c\left(t_{*}-t\right)^{2}}{a}}\right)\right]
$$

for all $t \in\left[0, t_{*}\right]$.

We note that the error term in (4.1) is small over most of the time period $\left[t, t_{*}\right]$, but it becomes order 1 when $t_{*}-t=O(\sqrt{a})$. After integration, Lemma 4.1 implies that

$$
\log \frac{I\left(t_{*}\right)}{I(0)}=\frac{3 \sqrt{N}}{2 \lambda_{*}} \log \left(t_{*}+1\right)+O\left(a^{-1 / 2}\right)=\frac{3 \sqrt{N}}{2 \lambda_{*}} \log \frac{a}{2 \sqrt{N}}+O\left(a^{-1 / 2}\right),
$$

where the error term is dominated by the region $t_{*}-t=O(\sqrt{a})$.

\section{The free contribution}

We first look at the putative main term in this period.

Lemma 4.2 There exist $c>0$ and $C>0$ so that for all $t \in\left[t_{-}, t_{*}\right]$, we have the asymptotics

$$
\int_{\mathbb{R}} \psi(x) p(t, x) \mathrm{d} x=A \gamma_{*} e^{-\gamma_{*} \bar{x}_{0}}(t+1)^{3 \sqrt{N} /\left(2 \lambda_{*}\right)} e^{-\sqrt{N} a}\left[1+O\left(e^{-\frac{c\left(t_{*}-t\right)^{2}}{a}}\right)\right]
$$

and

$$
\int_{\mathbb{R}} \psi^{\prime}(x) p(t, x) \mathrm{d} x=\sqrt{N} A \gamma_{*} e^{-\gamma_{*} \bar{x}_{0}}(t+1)^{3 \sqrt{N} /\left(2 \lambda_{*}\right)} e^{-\sqrt{N} a}\left[1+O\left(e^{-\frac{c\left(t_{*}-t\right)^{2}}{a}}\right)\right],
$$

as well as the error estimate

$$
\int_{\mathbb{R}}|E(t, x)| p(t, x) \psi(x) \mathrm{d} x \leq \frac{C}{t+1} e^{-\frac{c\left(t_{*}-t\right)^{2}}{a}} \int_{\mathbb{R}} \psi^{\prime}(x) p(t, x) \mathrm{d} x .
$$

Moreover, for all $t \in\left[t_{-}, t_{*}\right]$,

$$
I(t) \geq \int_{\mathbb{R}} \psi(x) p(t, x) \mathrm{d} x \geq c(t+1)^{3 \sqrt{N} /\left(2 \lambda_{*}\right)} e^{-\sqrt{N} a} .
$$

The first estimate in this lemma permits $\int_{\mathbb{R}} \psi p$ to reach 0 when $t_{*}-t=O(\sqrt{a})$, which could make $\dot{I}(t) / I(t)$ very large. The lower bound (4.6) ensures that this does not occur. 
Proof. To prove (4.3), let us first re-write $p(t, x)$ in a more convenient form, starting from (3.2):

$$
\begin{aligned}
p(t, x) & =\frac{1}{\sqrt{4 \pi t}} \exp \left\{-N t-\frac{1}{4 t}\left[x+a-\frac{3}{2 \lambda_{*}} \log (t+1)\right]^{2}\right\} \\
& =\frac{1}{\sqrt{4 \pi t}} \exp \left\{-\frac{1}{4 t}\left[x+a-\frac{3}{2 \lambda_{*}} \log (t+1)-2 \sqrt{N} t\right]^{2}-\sqrt{N}\left[x+a-\frac{3}{2 \lambda_{*}} \log (t+1)\right]\right\} \\
& =\frac{1}{\sqrt{4 \pi t}} e^{-a \sqrt{N}}(t+1)^{3 \sqrt{N} /\left(2 \lambda_{*}\right)} e^{-\sqrt{N} x} \exp \left\{-\frac{1}{4 t}[x-\nu(t)]^{2}\right\} .
\end{aligned}
$$

where we used the notation

$$
\nu(t)=-a+\frac{3}{2 \lambda_{*}} \log (t+1)+2 \sqrt{N} t
$$

It follows that

$$
\int_{\mathbb{R}} \psi(x) p(t, x) \mathrm{d} x=e^{-\sqrt{N} a}(t+1)^{3 \sqrt{N} /\left(2 \lambda_{*}\right)} \int_{\mathbb{R}} \zeta(x) \exp \left\{-\frac{1}{4 t}[x-\nu(t)]^{2}\right\} \frac{\mathrm{d} x}{\sqrt{4 \pi t}},
$$

with

$$
\zeta(x)=\psi(x) \exp (-\sqrt{N} x)
$$

Note that $(2.30)$ gives

$$
\zeta(x)=A \gamma_{*} e^{-\gamma_{*} \bar{x}_{0}} \mathbb{1}_{\mathbb{R}_{-}}(x)+O\left(e^{-c|x|}\right) .
$$

Note also that

$$
\nu(t)=-a+\frac{3}{2 \lambda_{*}} \log (t+1)+2 \sqrt{N}\left(t_{*}-\left(t_{*}-t\right)\right) \leq-2 \sqrt{N}\left(t_{*}-t\right)+C \log a .
$$

When $t_{*}-t \leq \sqrt{a}$, (4.3) follows simply from $0 \leq \zeta \leq C$, which is a consequence of (4.10) We may thus assume that $0 \leq t \leq t_{*}-\sqrt{a}$. Since $\log a \ll \sqrt{a}$ when $a$ is large, we can in turn assume that (4.11) yields

$$
\nu(t) \leq-c\left(t_{*}-t\right)
$$

Using (4.10) and (4.11), we can write the integral in the right side of (4.9) as

$$
\begin{aligned}
\int_{\mathbb{R}} \zeta(x) \exp \left\{-\frac{1}{4 t}[x-\nu(t)]^{2}\right\} \frac{\mathrm{d} x}{\sqrt{4 \pi t}}=A \gamma_{*} e^{-\gamma_{*} \bar{x}_{0}} \int_{\mathbb{R}_{-}} \exp \left\{-\frac{1}{4 t}[x-\nu(t)]^{2}\right\} \frac{\mathrm{d} x}{\sqrt{4 \pi t}} \\
+O\left(\int_{\mathbb{R}^{-c|x|}} \exp \left\{-\frac{1}{4 t}[x-\nu(t)]^{2}\right\} \frac{\mathrm{d} x}{\sqrt{4 \pi t}}\right) .
\end{aligned}
$$

We can write the main term on the right side as

$$
\begin{aligned}
\int_{\mathbb{R}_{-}} \exp \left\{-\frac{1}{4 t}[x-\nu(t)]^{2}\right\} \frac{\mathrm{d} x}{\sqrt{4 \pi t}} & =1-\int_{\mathbb{R}_{+}} \exp \left\{-\frac{1}{4 t}[x+|\nu(t)|]^{2}\right\} \frac{\mathrm{d} x}{\sqrt{4 \pi t}} \\
& =1+O\left(e^{-\nu(t)^{2} /(4 t)}\right)=1+O\left(e^{-\frac{c\left(t_{*}-t\right)^{2}}{a}}\right) .
\end{aligned}
$$

We used (4.12) and $t \leq a$ in the last step. To bound the error in (4.13), we break the integral at the position $\nu(t) / 2$. For $x \leq \nu(t) / 2$ we write, on the one hand,

$$
\int_{-\infty}^{\nu(t) / 2} e^{-c|x|} \exp \left\{-\frac{1}{4 t}[x-\nu(t)]^{2}\right\} \frac{\mathrm{d} x}{\sqrt{4 \pi t}} \leq \frac{1}{\sqrt{4 \pi t}} \int_{-\infty}^{\nu(t) / 2} e^{c x} \mathrm{~d} x=\frac{C}{\sqrt{t}} e^{c \nu(t) / 2}
$$


and, on the other hand

$$
\int_{-\infty}^{\nu(t) / 2} e^{-c|x|} \exp \left\{-\frac{1}{4 t}[x-\nu(t)]^{2}\right\} \frac{\mathrm{d} x}{\sqrt{4 \pi t}} \leq e^{c \nu(t) / 2} \int_{\mathbb{R}} \exp \left\{-\frac{1}{4 t}[x-\nu(t)]^{2}\right\} \frac{\mathrm{d} x}{\sqrt{4 \pi t}}=e^{c \nu(t) / 2}
$$

Combining these two bounds gives

$$
\begin{aligned}
\int_{-\infty}^{\nu(t) / 2} e^{-c|x|} \exp \left\{-\frac{1}{4 t}[x-\nu(t)]^{2}\right\} \frac{\mathrm{d} x}{\sqrt{4 \pi t}} \leq \frac{C}{\sqrt{1+t}} e^{c \nu(t) / 2} & \leq \frac{C}{\sqrt{1+t}} e^{-c\left(t_{*}-t\right)} \\
& \leq \frac{C}{\sqrt{1+t}} e^{-\frac{c\left(t_{*}-t\right)^{2}}{a}}
\end{aligned}
$$

For the the region $x \geq \nu(t) / 2$ of the integral in the error term in (4.13), we have

$$
\int_{\nu(t) / 2}^{\infty} e^{-c|x|} \exp \left\{-\frac{1}{4 t}[x-\nu(t)]^{2}\right\} \frac{\mathrm{d} x}{\sqrt{4 \pi t}} \leq \frac{C}{\sqrt{t}} e^{-\nu(t)^{2} /(16 t)} \leq \frac{C}{\sqrt{1+t}} e^{-\frac{c\left(t_{*}-t\right)^{2}}{a}} .
$$

In the last step, we wrote $\nu(t)^{2} /(16 t) \geq 1 /(32 t)+\nu(t)^{2} /(32 t)$ and used

$$
\frac{1}{\sqrt{t}} \exp \left(-\frac{1}{32 t}\right) \leq \frac{C}{\sqrt{t+1}}
$$

Now, (4.3) follows from (4.9), (4.13), (4.14), (4.15) and (4.16). The proof of (4.4) is identical: we just need to use $\tilde{\zeta}(x):=\psi^{\prime}(x) \exp (-\sqrt{N} x)=\sqrt{N} A \gamma_{*} e^{-\gamma_{*} \bar{x}_{0}} \mathbb{1}_{\mathbb{R}_{-}}(x)+O\left(e^{-c|x|}\right)$ in place of (4.10).

We now turn to (4.5). Recalling the definitions (2.34) of $E(t, x)$ and (2.19) of $V(t, x)$, and noting that $h^{\prime}$ is Lipschitz, we recall the following bound from $[10,17,18]$ :

$$
|E(t, x)| \leq C\left|U_{0}(x)-H(t, x+m(t))\right| \leq \frac{C e^{-c|x|}}{\sqrt{t+1}}
$$

Strictly speaking, [18] only proves this bound for $x \geq 0$. However, the wave $U_{0}$ is linearly stable on $\mathbb{R}_{-}$and (1.11) and (3.7) imply that $U_{0}-H$ decays exponentially in space. Because

$$
\left|U_{0}(0)-H(t, m(t))\right| \leq C(t+1)^{-1 / 2}
$$

at the boundary $x=0$ of $\mathbb{R}_{-}$, it is straightforward to control $\left|U_{0}-H\right|$ by a supersolution of the form $C(t+1)^{-1 / 2} e^{-c|x|}$ on $\mathbb{R}_{-}$, perhaps after translation. We omit the details.

Inserting this factor in (4.9) and recalling that $\zeta(x) \leq C$, we find

$$
\int_{\mathbb{R}}|E(t, x)| \psi(x) p(t, x) \mathrm{d} x \leq \frac{C}{\sqrt{t+1}} e^{-\sqrt{N} a}(t+1)^{3 \sqrt{N} /\left(2 \lambda_{*}\right)} \int_{\mathbb{R}} e^{-c|x|} \exp \left\{-\frac{1}{4 t}[x+|\nu(t)|]^{2}\right\} \frac{\mathrm{d} x}{\sqrt{4 \pi t}} .
$$

We estimated this integral integral above, assuming $t_{*}-t \geq \sqrt{a}$. Using (4.15) and (4.16), we obtain (4.5) for such times. When $t_{*}-t \leq \sqrt{a}$, we can bound the Gaussian in the above integral by 1 to obtain (4.5).

To obtain the lower bound in (4.6), we first recall (3.4), which implies $r \geq p$ and $I \geq \int_{\mathbb{R}} \psi p$. Next, we note that $\zeta \geq c \mathbb{1}_{\mathbb{R}_{-}}$. So, (4.9) implies

$$
I(t) \geq \int_{\mathbb{R}} \psi(x) p(t, x) \mathrm{d} x \geq c(t+1)^{3 \sqrt{N} /\left(2 \lambda_{*}\right)} e^{-\sqrt{N} a} \int_{\mathbb{R}_{-}} \exp \left\{-\frac{1}{4 t}[x-\nu(t)]^{2}\right\} \frac{\mathrm{d} x}{\sqrt{4 \pi t}} .
$$


Changing variables via $\eta=\frac{x-\nu(t)}{\sqrt{t}}$, we have

$$
\int_{\mathbb{R}_{-}} \exp \left\{-\frac{1}{4 t}[x-\nu(t)]^{2}\right\} \frac{\mathrm{d} x}{\sqrt{4 \pi t}}=\int_{-\infty}^{-\nu(t) / \sqrt{t}} e^{-\eta^{2} / 4} \frac{\mathrm{d} \eta}{\sqrt{4 \pi}} .
$$

By (4.11), for $t \geq t_{-}$,

$$
-\frac{\nu(t)}{\sqrt{t}} \geq-\frac{C \log a}{\sqrt{a}} \geq-1,
$$

assuming $a$ is large. In light of (4.19), (4.18) yields

$$
I(t) \geq \int_{\mathbb{R}} \psi(x) p(t, x) \mathrm{d} x \geq c(t+1)^{3 \sqrt{N} /\left(2 \lambda_{*}\right)} e^{-\sqrt{N} a} .
$$

This finishes the proof of Lemma 4.2.

\section{The main corrector contribution}

Next, we control the contributions of $q_{\mathrm{m}}$ to $I(t)$ and $\dot{I}(t)$. We only need to consider $t \geq t_{-}$as $q_{\mathrm{m}} \equiv 0$ for $t<t_{-}$.

Lemma 4.3 There exist $c>0$ and $C>0$ such that for all $t \in\left[t_{-}, t_{*}\right]$,

$$
\int_{\mathbb{R}}\left[\psi(x)+\psi^{\prime}(x)\right] q_{\mathrm{m}}(t, x) \mathrm{d} x \leq C(t+1)^{3 \sqrt{N} /\left(2 \lambda_{*}\right)} e^{-\sqrt{N} a} e^{-\frac{c\left(t_{*}-t\right)^{2}}{a}}
$$

and

$$
\int_{\mathbb{R}}|E(t, x)| \psi(x) q_{\mathrm{m}}(t, x) \mathrm{d} x \leq \frac{C}{a}(t+1)^{3 \sqrt{N} /\left(2 \lambda_{*}\right)} e^{-\sqrt{N} a} e^{-\frac{c\left(t_{*}-t\right)^{2}}{a}} .
$$

Recall that $q_{\mathrm{m}}$ satisfies (3.15):

$$
\partial_{t} q_{\mathrm{m}}=\partial_{x}^{2} q_{\mathrm{m}}-\frac{3}{2 \lambda_{*}(t+1)} \partial_{x} q_{\mathrm{m}}-V(t, x) q_{\mathrm{m}}+(N-V(t, x)) \mathbb{1}_{\left[t_{-}, \infty\right)}(t) p, \quad q_{\mathrm{m}}(0, \cdot)=0 .
$$

We use the Duhamel formula

$$
q_{\mathrm{m}}(t, x)=\int_{t_{-}}^{t} q^{s}(t, x) \mathrm{d} s
$$

to control $q_{\mathrm{m}}$. Here, for $s \geq t_{-}$, the function $q^{s}$ satisfies

$$
\begin{aligned}
& \partial_{t} q^{s}=\partial_{x}^{2} q^{s}-\frac{3}{2 \lambda_{*}(t+1)} \partial_{x} q^{s}-V(t, x) q^{s}, t>s, \\
& q^{s}(s, x)=[N-V(s, x)] p(s, x) .
\end{aligned}
$$

We can control this for all $t_{-} \leq s \leq t$.

Lemma 4.4 For all $s \geq t_{-}$and $t \geq s$,

$$
\begin{aligned}
& \int_{\mathbb{R}} \psi(x) q^{s}(t, x) \mathrm{d} x \leq C \frac{s^{2}}{a^{2}} \Lambda(s ; a), \\
& \int_{\mathbb{R}} \psi^{\prime}(x) q^{s}(t, x) \mathrm{d} x \leq C \frac{s^{2}}{a^{2}} \Lambda(s ; a)(t-s+1)^{-1 / 2}, \\
& \int_{\mathbb{R}}|E(t, x)| \psi(x) q^{s}(t, x) \mathrm{d} x \leq C \frac{s^{2}}{a^{2}} \Lambda(s ; a)(t+1)^{-1 / 2}(t-s+1)^{-3 / 2} .
\end{aligned}
$$


Proof. We first get rid of the logarithmic term in (3.11) and (3.13). We claim that

$$
\begin{aligned}
p(s, x) & =\Lambda(s ; a) e^{-a x /(2 s)} \exp \left[-\frac{1}{4 s}\left(x-\frac{3}{2 \lambda_{*}} \log (s+1)\right)^{2}\right] \\
& \leq C \Lambda(s ; a) e^{-a x /(2 s)} \exp \left(-\frac{x^{2}}{8 s}\right) .
\end{aligned}
$$

After all, an elementary study of the quadratic polynomial shows that for all $\alpha>0$ and $\varepsilon \in(0,1)$, there there exists $C$ depending on $\alpha$ and $\varepsilon$ such that

$$
\frac{[z+\alpha \log (t+1)]^{2}}{4 t} \geq(1-\varepsilon) \frac{z^{2}}{4 t}-C \quad \text { for all } z \in \mathbb{R} \text { and } t>0 .
$$

By Lemma 3.1, we obtain

$$
\Lambda(s ; a)^{-1} q^{s}(s, x)=\Lambda(s ; a)^{-1}[N-V(s, x)] p(s, x) \leq \begin{cases}C \exp \left[-\left(\frac{a}{2 s}-\gamma_{*}\right) x\right] & \text { for } x<0 \\ C \exp \left(-\frac{a x}{2 s}\right) \exp \left(-\frac{x^{2}}{8 s}\right) & \text { for } x \geq 0\end{cases}
$$

Since $s \geq t_{-}$, we have

$$
\frac{a}{2 s}-\gamma_{*} \leq \frac{1}{2 \xi_{-}}-\gamma_{*}=: \kappa_{-} \in(0, \sqrt{N}) .
$$

Let us fix $\alpha \in\left(\kappa_{-}, \sqrt{N}\right)$. By Lemma 3.1, there exists $K \geq 0$ such that

$$
V(t, x) \geq \alpha^{2} \mathbb{1}_{(-\infty,-K)}(x) .
$$

We see from (4.26) and (4.28) that $q^{s} /[C \Lambda(s ; a)]$ satisfies the hypotheses of Lemma 3.3, with $\kappa_{-}$ given in (4.27) and $\kappa_{+}=a /(2 s)$. The bounds in (4.24) follow from Lemma 3.3 and $s \geq t_{-}$.

We can now control $q_{\mathrm{m}}$ in terms of $q^{s}$.

Proof of Lemma 4.3. In light of (4.22), we need to integrate (4.24) over $s \in\left[t_{-}, t\right]$ for $t \in\left[t_{-}, t_{*}\right]$. For $s \geq t_{-}$, the definition (3.12) of $\Lambda$ implies

$$
\Lambda(s ; a) \leq \frac{C}{\sqrt{a}}(s+1)^{3 a /\left(4 \lambda_{*} s\right)} e^{-a \theta(s / a)}
$$

for the rate function

$$
\theta(\xi)=N \xi+\frac{1}{4 \xi} \quad \text { for } \xi>0
$$

This strictly convex function is minimized at $\xi_{*}=1 /(2 \sqrt{N})$, so there exists $c>0$ such that

$$
\theta(\xi)=N \xi+\frac{1}{4 \xi} \geq \theta\left(\xi_{*}\right)+c\left(\xi-\xi_{*}\right)^{2}=\sqrt{N}+c\left(\xi-\xi_{*}\right)^{2}
$$

for all $\xi \in(0,1]$. Therefore,

$$
\Lambda(s ; a) \leq \frac{C}{\sqrt{a}}(s+1)^{3 a /\left(4 \lambda_{*} s\right)} e^{-\sqrt{N} a-\frac{c\left(t_{*}-s\right)^{2}}{a}}
$$

for all $s \in\left[t_{-}, a\right]$.

We next handle the polynomial prefactor, which we write as

$$
(s+1)^{3 a /\left(4 \lambda_{*} s\right)}=\exp \left[\frac{3 a}{4 \lambda_{*} s} \log (s+1)\right] .
$$


For any $\varepsilon>0$, we employ the Peter-Paul inequality $2 A B \leq \varepsilon A^{2}+B^{2} / \varepsilon$ :

$$
a\left(\frac{1}{s}-\frac{1}{t_{*}}\right) \log (s+1) \leq \frac{C\left(t_{*}-s\right) \log a}{a} \leq \frac{\varepsilon\left(t_{*}-s\right)^{2}}{a}+\frac{C^{2} \log ^{2} a}{4 \varepsilon a} \leq \frac{\varepsilon\left(t_{*}-s\right)^{2}}{a}+C_{\varepsilon} .
$$

Exponentiating, this implies that

$$
(s+1)^{3 a /\left(4 \lambda_{*} s\right)} \leq C_{\varepsilon}(s+1)^{3 a /\left(4 \lambda_{*} t_{*}\right)} e^{\frac{\varepsilon\left(t_{*}-s\right)^{2}}{a}}=C_{\varepsilon}(s+1)^{3 \sqrt{N} /\left(2 \lambda_{*}\right)} e^{\frac{\varepsilon\left(t_{*}-s\right)^{2}}{a}} .
$$

Taking $\varepsilon \ll 1$, we can absorb the last factor into the Gaussian term in (4.30). Thus, (4.30) and (4.31) yield

$$
\Lambda(s ; a) \leq \frac{C}{\sqrt{a}}(s+1)^{3 \sqrt{N} /\left(2 \lambda_{*}\right)} e^{-\sqrt{N} a-\frac{c\left(t_{*}-s\right)^{2}}{a}} \leq \frac{C}{\sqrt{a}}(s+1)^{3 \sqrt{N} /\left(2 \lambda_{*}\right)} e^{-\sqrt{N} a-\frac{c\left(t_{*}-s\right)^{2}}{a}}
$$

for all $s \in\left[t_{-}, a\right]$. We now combine (4.24) and (4.32) to control the contribution of $q_{\mathrm{m}}$. At these times, we do not need the distinction between $\psi$ and $\psi^{\prime}$ in (4.24):

$$
\int_{\mathbb{R}}\left[\psi(x)+\psi^{\prime}(x)\right] q_{\mathrm{m}}(t, x) \mathrm{d} x=\int_{t_{-}}^{t} \int_{\mathbb{R}}\left[\psi(x)+\psi^{\prime}(x)\right] q^{s}(t, x) \mathrm{d} x \mathrm{~d} s \leq C \int_{t_{-}}^{t} \Lambda(s ; a) \mathrm{d} s .
$$

Using (4.32) and changing variables via $\eta=\frac{t_{*}-s}{\sqrt{a}}$, we obtain

$$
\begin{aligned}
\int_{\mathbb{R}}\left[\psi(x)+\psi^{\prime}(x)\right] q_{\mathrm{m}}(t, x) \mathrm{d} x & \leq C(t+1)^{3 \sqrt{N} /\left(2 \lambda_{*}\right)} e^{-\sqrt{N} a} \int_{\left(t_{*}-t\right) / \sqrt{a}}^{\infty} e^{-c \eta^{2}} \mathrm{~d} \eta \\
& \leq C(t+1)^{3 \sqrt{N} /\left(2 \lambda_{*}\right)} e^{-\sqrt{N} a} e^{-\frac{c\left(t_{*}-t\right)^{2}}{a}} .
\end{aligned}
$$

This is (4.20). Finally, the third line in (4.24) and (4.32) imply:

$$
\int_{\mathbb{R}}|E(t, x)| \psi(x) q_{\mathrm{m}}(t, x) \mathrm{d} x \leq \frac{C}{a}(t+1)^{3 \sqrt{N} /\left(2 \lambda_{*}\right)} e^{-\sqrt{N} a} \int_{t_{-}}^{t}(t-s+1)^{-3 / 2} e^{-c\left(t_{*}-s\right)^{2} / a} \mathrm{~d} s .
$$

Since $(|y|+1)^{-3 / 2}$ is integrable, the integral on the right side of $(4.33)$ is bounded by $C e^{-\left(t_{*}-t\right)^{2} / a}$. Hence

$$
\int_{\mathbb{R}}|E(t, x)| \psi(x) q_{\mathrm{m}}(t, x) \mathrm{d} x \leq \frac{C}{a}(t+1)^{3 \sqrt{N} /\left(2 \lambda_{*}\right)} e^{-\sqrt{N} a} e^{-c\left(t_{*}-t\right)^{2} / a} .
$$

We have thus verified (4.21) and completed the proof of Lemma 4.3.

\section{The proof of Lemma 4.1}

We wish to understand $I(t)$ and $\dot{I}(t)$ given by (2.32) and (2.35), respectively. Recall that $r=$ $p+q_{\mathrm{e}}+q_{\mathrm{m}}$. We use Lemmas $4.2,3.2$, and 4.3 to control the terms with $p, q_{\mathrm{e}}$, and $q_{\mathrm{m}}$, respectively. These lemmas yield

$$
I(t)=\int_{\mathbb{R}} \psi(x) r(t, x) \mathrm{d} x=A \gamma_{*} e^{-\gamma_{*} \bar{x}_{0}}(t+1)^{3 \sqrt{N} /\left(2 \lambda_{*}\right)} e^{-\sqrt{N} a}\left[1+O\left(e^{-\frac{c\left(t_{*}-t\right)^{2}}{a}}\right)\right]
$$

and

$$
\begin{aligned}
\frac{\mathrm{d} I}{\mathrm{~d} t}(t) & =\frac{3}{2 \lambda_{*}(t+1)} \int_{\mathbb{R}} \psi^{\prime}(x) r(t, x) \mathrm{d} x+\int_{\mathbb{R}} E(t, x) \psi(x) r(t, x) \mathrm{d} x \\
& =\frac{3}{2 \lambda_{*}(t+1)} \sqrt{N} A \gamma_{*} e^{-\gamma_{*} \bar{x}_{0}}(t+1)^{3 \sqrt{N} /\left(2 \lambda_{*}\right)} e^{-\sqrt{N} a}\left[1+O\left(e^{-\frac{c\left(t_{*}-t\right)^{2}}{a}}\right)\right] .
\end{aligned}
$$


Finally, the lower bound (4.6) and (3.4) imply that $\dot{I}(t) / I(t)$ doesn't become singular as $t \rightarrow t_{*}$. Therefore

$$
\frac{\dot{I}(t)}{I(t)}=\frac{3 \sqrt{N}}{2 \lambda_{*}(t+1)}\left[1+O\left(e^{-\frac{c\left(t_{*}-t\right)^{2}}{a}}\right)\right]
$$

when $t \in\left[0, t_{*}\right]$, and the proof of Lemma 4.1 is complete.

\section{$5 \quad$ Middle times}

Here, we consider the time interval $t \in\left[t_{*}, a\right]$. Now, the story changes: the main corrector $q_{\mathrm{m}}(t, x)$ becomes the dominant term in $I(t)$ and $\dot{I}(t)$, though, of course, the homogeneous term $p(t, x)$ is comparable to it when $t-t_{*}=O(\sqrt{a})$. Again, Lemma 3.2 shows that the contributions of $q_{\mathrm{e}}$ are negligible relative to those of $q_{\mathrm{m}}$ in Lemma 5.3 below.

We will prove the following.

Lemma 5.1 There exist $c>0$ and $C>0$ such that for all $t \in\left[t_{*}, a\right]$,

$$
\frac{|\dot{I}(t)|}{I(t)} \leq \frac{C}{a}\left[\left(t-t_{*}+1\right)^{-1 / 2}+e^{-c\left(t-t_{*}\right)^{2} / a}\right] .
$$

After integration, Lemma 5.1 implies that

$$
\log \frac{I(a)}{I\left(t_{*}\right)}=O\left(a^{-1 / 2}\right) .
$$

\section{The free contribution}

We first bound the contributions of the free term $p$.

Lemma 5.2 There exist $c>0$ and $C>0$ such that for all $t \in\left[t_{*}, a\right]$,

$$
\int_{\mathbb{R}}\left[\psi(x)+\psi^{\prime}(x)\right] p(t, x) \mathrm{d} x \leq C(t+1)^{3 \sqrt{N} /\left(2 \lambda_{*}\right)} e^{-\sqrt{N} a} e^{-\frac{c\left(t-t_{*}\right)^{2}}{a}}
$$

and

$$
\int_{\mathbb{R}}|E(t, x)| p(t, x) \mathrm{d} x \leq \frac{C}{a}(t+1)^{3 \sqrt{N} /\left(2 \lambda_{*}\right)} e^{-\sqrt{N} a} e^{-\frac{c\left(t-t_{*}\right)^{2}}{a}} .
$$

Proof. Recall expression (3.11) for $p(t, x)$. We consider $\left(\psi+\psi^{\prime}\right) p$ separately on $\mathbb{R}_{ \pm}$. When $x \leq 0$, we use $\psi(x)+\psi^{\prime}(x) \leq C \exp (\sqrt{N} x)$ from (2.30), and obtain

$$
\left[\psi(x)+\psi^{\prime}(x)\right] p(t, x) \leq C \Lambda(t ; a) \exp \left[\left(\sqrt{N}-\frac{a}{2 t}\right) x\right] g(t, x) \quad \text { for } x \leq 0 .
$$

Now $t \geq t_{*}$, so $\sqrt{N}-a /(2 t) \geq 0$. Also, (3.13) shows that $\int_{\mathbb{R}} g(t, x) \mathrm{d} x=\sqrt{4 \pi t}$. It follows that

$$
\int_{\mathbb{R}_{-}}\left[\psi(x)+\psi^{\prime}(x)\right] p(t, x) \mathrm{d} x \leq C \Lambda(t ; a) \int_{\mathbb{R}} g(t, x) \mathrm{d} x \leq C \sqrt{t} \Lambda(t ; a) \quad \text { for all } t \geq t_{*} .
$$

When $x \geq 0$, we use $\psi(x)+\psi^{\prime}(x) \leq C(1+x)$ from (2.30) and $t \leq a$ to obtain

$$
\left[\psi(x)+\psi^{\prime}(x)\right] p(t, x) \leq C(1+x) \Lambda(t ; a) e^{-a x /(2 t)} \leq C(1+x) \Lambda(t ; a) e^{-x / 2} \quad \text { for } x \geq 0 .
$$


So

$$
\int_{\mathbb{R}_{+}}\left[\psi(x)+\psi^{\prime}(x)\right] p(t, x) \mathrm{d} x \leq C \Lambda(t ; a) .
$$

We now turn to the error term with $E$. Recalling the bound (4.17) on $E$, (5.3) and (5.5) imply

$$
\int_{\mathbb{R}}|E(t, x)| \psi(x) p(t, x) \mathrm{d} x \leq \frac{C \Lambda(t ; a)}{\sqrt{t+1}} \int_{\mathbb{R}} e^{-c|x|} \mathrm{d} x \leq \frac{C \Lambda(t ; a)}{\sqrt{t}} .
$$

The lemma follows from (5.4), (5.6) and (5.7) using (4.30) with $a / t \leq a / t_{*}=2 \sqrt{N}$.

\section{The main corrector contribution}

We now estimate the contribution of $q_{\mathrm{m}}(t, x)$ on the interval $\left[t_{*}, a\right]$.

Lemma 5.3 There exist $c>0$ and $C>0$ such that for all $t \in\left[t_{*}, a\right]$,

$$
\begin{aligned}
\int \psi^{\prime}(x) q_{\mathrm{m}}(t, x) \mathrm{d} x & \leq C(t+1)^{3 \sqrt{N} /\left(2 \lambda_{*}\right)} e^{-\sqrt{N} a}\left[\left(t-t_{*}+1\right)^{-1 / 2}+e^{-c\left(t-t_{*}\right)^{2} / a}\right], \\
\int|E(t, x)| \psi(x) q_{\mathrm{m}}(t, x) \mathrm{d} x & \leq \frac{C}{a}(t+1)^{3 \sqrt{N} /\left(2 \lambda_{*}\right)} e^{-\sqrt{N} a}\left[\left(t-t_{*}+1\right)^{-1 / 2}+e^{-c\left(t-t_{*}\right)^{2} / a}\right] .
\end{aligned}
$$

Moreover, for all $t \geq t_{*}$,

$$
I(t) \geq \int_{\mathbb{R}} \psi(x) q_{\mathrm{m}}(t, x) \mathrm{d} x \geq c a^{3 \sqrt{N} /\left(2 \lambda_{*}\right)} e^{-\sqrt{N} a} .
$$

We emphasize that the last bound holds for all $t \geq t_{*}$, and thus extends to the late times after $a$ as well.

Proof. We again represent $q_{\mathrm{m}}(t, x)$ via the Duhamel formula (4.22) as an integral of $q^{s}$ satisfying (4.23). By Lemma 4.4,

$$
\begin{aligned}
& \int_{\mathbb{R}} \psi^{\prime}(x) q_{\mathrm{m}}(t, x) \mathrm{d} x \leq C \int_{t_{-}}^{t} \Lambda(s ; a)(t-s+1)^{-1 / 2} \mathrm{~d} s \\
& \int_{\mathbb{R}}|E(t, x)| \psi(x) q_{\mathrm{m}}(t, x) \mathrm{d} x \leq C \int_{t_{-}}^{t} \Lambda(s ; a) a^{-1 / 2}(t-s+1)^{-3 / 2} \mathrm{~d} s .
\end{aligned}
$$

By (4.32),

$$
\Lambda(s ; a) \leq \frac{C}{\sqrt{a}}(t+1)^{3 \sqrt{N} /\left(2 \lambda_{*}\right)} e^{-\sqrt{N} a} e^{-c\left(s-t_{*}\right)^{2} / a}
$$

for $t_{-} \leq s \leq t \leq a$. In light of (5.11), we must bound integrals of the form

$$
Z_{\alpha}(t):=\int_{t_{-}}^{t}(t-s+1)^{-\alpha} e^{-c\left(s-t_{*}\right)^{2} / a} \mathrm{~d} s \leq \int_{0}^{\infty}(s+1)^{-\alpha} e^{-c\left(t-t_{*}-s\right)^{2} / a} \mathrm{~d} s
$$

for $\alpha \in\{1 / 2,3 / 2\}$.

We cut the integral in the right side of $(5.13)$ at $s=\left(t-t_{*}\right) / 2$ :

$$
\int_{\frac{t-t_{*}}{2}}^{\infty}(s+1)^{-\alpha} e^{-c\left(t-t_{*}-s\right)^{2} / a} \mathrm{~d} s \leq\left(\frac{t-t_{*}}{2}+1\right)^{-\alpha} \int_{\frac{t-t_{*}}{2}}^{\infty} e^{-c\left(t-t_{*}-s\right)^{2} / a} \mathrm{~d} s \leq C \sqrt{a}\left(t-t_{*}+1\right)^{-\alpha}
$$


and

$$
\begin{aligned}
\int_{0}^{\frac{t-t_{*}}{2}}(s+1)^{-\alpha} e^{-c\left(t-t_{*}-s\right)^{2} / a} \mathrm{~d} s \leq \int_{0}^{\frac{t-t_{*}}{2}} e^{-c\left(t-t_{*}-s\right)^{2} / a} \mathrm{~d} s & \leq \int_{\frac{t-t_{*}}{2}}^{\infty} e^{-c r^{2} / a} \mathrm{~d} r \\
& \leq C \sqrt{a} e^{-c\left(t-t_{*}\right)^{2} /(4 a)} .
\end{aligned}
$$

we conclude that

$$
Z_{\alpha}(t) \leq C \sqrt{a}\left[\left(t-t_{*}+1\right)^{-\alpha}+e^{-c\left(t-t_{*}\right)^{2} / a}\right] .
$$

When $\alpha>1$, the integrability of $(s+1)^{-\alpha}$ gives an alternative bound. Still cutting at $s=\left(t-t_{*}\right) / 2$, we now write

$$
\int_{\frac{t-t_{*}}{2}}^{\infty}(s+1)^{-\alpha} e^{-c\left(t-t_{*}-s\right)^{2} / a} \mathrm{~d} s \leq \int_{\frac{t-t_{*}}{2}}^{\infty}(s+1)^{-\alpha} \mathrm{d} s \leq C\left(t-t_{*}+1\right)^{1-\alpha}
$$

and

$$
\int_{0}^{\frac{t-t_{*}}{2}}(s+1)^{-\alpha} e^{-c\left(t-t_{*}-s\right)^{2} / a} \mathrm{~d} s \leq e^{-c\left(t-t_{*}\right)^{2} /(4 a)} \int_{0}^{\infty}(s+1)^{-\alpha} \mathrm{d} s \leq C e^{-c\left(t-t_{*}\right)^{2} /(4 a)} .
$$

We conclude that

$$
Z_{\alpha}(t) \leq C\left[\left(t-t_{*}+1\right)^{1-\alpha}+e^{-c\left(t-t_{*}\right)^{2} / a}\right] \quad \text { if } \alpha>1 .
$$

Combining (5.11), (5.12), and (5.14) with $\alpha=1 / 2$ gives

$$
\begin{aligned}
\int_{\mathbb{R}} \psi^{\prime}(x) q_{\mathrm{m}}(t, x) \mathrm{d} x & \leq \frac{C}{\sqrt{a}}(t+1)^{3 \sqrt{N} /\left(2 \lambda_{*}\right)} e^{-\sqrt{N} a} Z_{1 / 2}(t) \\
& \leq C(t+1)^{3 \sqrt{N} /\left(2 \lambda_{*}\right)} e^{-\sqrt{N} a}\left[\left(t-t_{*}+1\right)^{-1 / 2}+e^{-c\left(t-t_{*}\right)^{2} / a}\right],
\end{aligned}
$$

while (5.11), (5.12), and (5.15) with $\alpha=3 / 2$ imply

$$
\begin{aligned}
\int_{\mathbb{R}}|E(t, x)| \psi(x) q_{\mathrm{m}}(t, x) \mathrm{d} x & \leq \frac{C}{a}(t+1)^{3 \sqrt{N} /\left(2 \lambda_{*}\right)} e^{-\sqrt{N} a} Z_{3 / 2}(t) \\
& \leq \frac{C}{a}(t+1)^{3 \sqrt{N} /\left(2 \lambda_{*}\right)} e^{-\sqrt{N} a}\left[\left(t-t_{*}+1\right)^{-1 / 2}+e^{-c\left(t-t_{*}\right)^{2} / a}\right] .
\end{aligned}
$$

We have thus confirmed (5.8) and (5.9).

Finally, we need a lower bound on $I(t)$. With $p \geq 0$ and $q_{\mathrm{e}} \geq 0$, we can write for $t \geq t_{*}$

$$
I(t) \geq \int_{\mathbb{R}} q_{\mathrm{m}}(t, x) \psi(x) \mathrm{d} x=\int_{t_{-}}^{t} \int_{\mathbb{R}} q^{s}(t, x) \psi(x) \mathrm{d} x \mathrm{~d} s \geq \int_{t_{-}}^{t_{*}} \int_{\mathbb{R}_{+}} q^{s}(t, x) \psi(x) \mathrm{d} x \mathrm{~d} s,
$$

where we used again the Duhamel formula (4.22) and $q^{s} \geq 0$. We recall that $q^{s}$ satisfies (4.23). We need a lower bound on $q^{s}$, so we look for a subsolution to $(4.23)$ on $\mathbb{R}_{+}$.

We first focus on the initial condition $q^{s}(s, x)=[N-V(s, x)] p(s, x)$ for $x \in \mathbb{R}_{+}$. Recall the definition (2.19) of $V$. The comparison principle implies that $H$ is decreasing in $x$, so

$$
H(s, x+m(s)) \leq H(s, m(s)) \text { for all } x \geq 0 .
$$

By (1.9) and $s \geq t_{-}=\xi_{-} a, H(s, x+m(s))$ is very close to $U_{0}(0)<1$ provided $a$ is sufficiently large. We can thus assume that $H(s, m(s)) \leq 1-c$ for all $s \geq t_{-}$. Using (5.17) and (1.19), it follows that $N-V(s, x) \geq c$ in this region. That is,

$$
q^{s}(s, x) \geq c p(s, x) \quad \text { for all } s \geq t_{-}, x \geq 0 .
$$


Going back to (3.11), we see that for all $x \geq 0$ and $s \geq t_{-}$, we have

$$
p(s, x) \geq \Lambda(s ; a) e^{-C x} \exp \left\{-\frac{1}{4 s}\left[x-\frac{3}{2 \lambda_{*}} \log (s+1)\right]^{2}\right\} .
$$

We are free to assume $s \geq t_{-} \geq 2$, so a variation on (4.25) yields

$$
\frac{1}{4 s}\left[x-\frac{3}{2 \lambda_{*}} \log (s+1)\right]^{2} \leq \frac{x^{2}}{8(1-\varepsilon)}+C(\varepsilon) \quad \text { for all } s \geq t_{-}, x \in \mathbb{R}
$$

and $\varepsilon \in(0,1)$. Hence (5.19) and $e^{-C x} \geq C(\varepsilon) e^{-\varepsilon x^{2}}$ implies

$$
p(s, x) \geq c \Lambda(s ; a) e^{-C x} e^{-x^{2} / 7} \geq c \Lambda(s ; a) e^{-x^{2} / 6} \geq c \Lambda(s ; a) x e^{-x^{2} / 4},
$$

where we have allowed $c>0$ to change from expression to expression. We now define

$$
\varphi(\lambda, x)=\frac{x}{\lambda^{3 / 2}} \exp \left(-\frac{x^{2}}{4 \lambda}\right)
$$

and note for later reference that

$$
\int_{\mathbb{R}_{+}} x \varphi(\lambda, x) \mathrm{d} x=2 \sqrt{\pi} \text { for all } \lambda>0 .
$$

Combining (5.18) and (5.20), we can write

$$
q^{s}(s, x) \geq c \Lambda(s ; a) \varphi(1, x) \text { for all } s \geq t_{-}, x \geq 0 .
$$

Now, we consider the PDE in (4.23). Since we are looking for a lower bound on $q^{s}(t, x)$, we cannot neglect the negative term $-V(t, x) q^{s}$ in the right side of (4.23). By Lemma 3.1,

$$
V(t, x) \leq B e^{-c x}
$$

for all $t>0$ and $x>0$. To obtain a subsolution, we are free to impose a Dirichlet condition at $x=0$. We let $\underline{v}^{s}(t, x)$ solve

$$
\begin{aligned}
& \partial_{t} \underline{v}^{s}=\partial_{x}^{2} \underline{v}^{s}-\frac{3}{2 \lambda_{*}(t+1)} \partial_{x} \underline{v}^{s}-B e^{-c x} \underline{v}^{s} \quad \text { for } t>s \text { and } x>0, \\
& \underline{v}^{s}(t, 0)=0 \text { for } t>s, \\
& \underline{v}^{s}(s, x)=\varphi(1, x) \quad \text { for } x>0 .
\end{aligned}
$$

By (5.22) and (5.23), we have

$$
q^{s}(t, x) \geq c \Lambda(s ; a) \underline{v}^{s}(t, x) \quad \text { for all } t \geq s \geq t_{-}, x \geq 0 .
$$

Then, with (5.16),

$$
I(t) \geq c \int_{t_{-}}^{t_{*}} \Lambda(s ; a) \int_{\mathbb{R}_{+}} \underline{v}^{s}(t, x) \psi(x) \mathrm{d} x \mathrm{~d} s .
$$

The following lemma gives a lower bound on $\underline{v}^{s}(t, x)$.

Lemma 5.4 There exists $c>0$ such that for all $t \geq s$ and $x>0$ we have

$$
\underline{v}^{s}(t, x) \geq c \varphi(t-s+1, x)+\underline{R}(t, s, x)
$$

with

$$
|\underline{R}(t, s, x)| \leq C(t-s+1)^{-2} x e^{-x^{2} /[8(t-s+1)]}=C(t-s+1)^{-1 / 2} \varphi(2(t-s+1), x) .
$$


Proof. This is nearly Lemma 2.2 in [11]. The only difference is the exponentially decaying potential, which is negligible on the scale $x \sim \sqrt{t}$, where the analysis really happens.

Using (2.30), we have $\psi(x) \geq c x$ for $x \geq 0$. Then (5.25), (5.26), and (5.21) imply

$$
\begin{aligned}
\int_{\mathbb{R}_{+}} \underline{v}^{s}(t, x) \psi(x) \mathrm{d} x & \geq c \int_{\mathbb{R}_{+}} x \varphi(t-s+1, x) \mathrm{d} x-C(t-s+1)^{-1 / 2} \int_{\mathbb{R}} x \varphi(2(t-s+1), x) \mathrm{d} x \\
& \geq c-C(t-s+1)^{-1 / 2},
\end{aligned}
$$

where we have allowed $c$ to change from expression to expression. So $\int_{\mathbb{R}_{+}} \underline{v}^{s} \psi$ is uniformly positive once $t-s \geq C^{\prime}$ for $C^{\prime}$ large. On the other hand, $\underline{v}^{s}$ is positive, so the integral is positive on the time interval $\left[s, s+C^{\prime}\right]$. By compactness, it follows that

$$
\int_{\mathbb{R}_{+}} \underline{v}^{s}(t, x) \psi(x) \mathrm{d} x \geq c \quad \text { for all } t \geq s \geq t_{-} .
$$

Now (5.24) yields

$$
I(t) \geq c \int_{t_{-}}^{t_{*}} \Lambda(s ; a) \mathrm{d} s
$$

for all $t \geq t_{*}$. For $s \in\left[t_{-}, t_{*}\right],(3.12)$ implies

$$
\Lambda(s ; a) \geq \frac{c}{\sqrt{a}} a^{3 \sqrt{N} /\left(2 \lambda_{*}\right)} e^{-a \theta(s / a)}
$$

for $\theta(\xi)=N \xi+1 /(4 \xi)$. We recall that $\theta$ is strictly convex and attains its minimum of $\sqrt{N}$ at $\xi_{*}=1 /(2 \sqrt{N})=t_{*} / a$. Also, $t_{-}=\xi_{-} a$. Since $\theta$ is smooth on the interval $\left[\xi_{-}, \xi_{*}\right]$, there exists $C>0$ such that

$$
\theta(\xi) \leq \sqrt{N}+C\left(\xi-\xi_{*}\right)^{2} \quad \text { for all } \xi \in\left[\xi_{-}, \xi_{*}\right] .
$$

Then (5.28) yields

$$
\Lambda(s ; a) \geq \frac{c}{\sqrt{a}} a^{3 \sqrt{N} /\left(2 \lambda_{*}\right)} e^{-\sqrt{N} a} e^{-C\left(t_{*}-s\right)^{2} / a}
$$

and (5.27) implies

$$
I(t) \geq c a^{3 \sqrt{N} /\left(2 \lambda_{*}\right)} e^{-\sqrt{N} a} \int_{t_{-}}^{t_{*}} e^{-C\left(t_{*}-s\right)^{2} / a} \frac{\mathrm{d} s}{\sqrt{a}} \geq c a^{3 \sqrt{N} /\left(2 \lambda_{*}\right)} e^{-\sqrt{N} a}
$$

for all $t \geq t_{*}$. This completes the proof of Lemma 5.3.

\section{The proof of Lemma 5.1}

Gathering together Lemmas 3.2, 5.2, and 5.3, we obtain

$$
\begin{aligned}
|\dot{I}(t)| & \leq \frac{3}{2(t+1)} \int_{\mathbb{R}} \psi^{\prime}(x) r(t, x) \mathrm{d} x+\int_{\mathbb{R}}|E(t, x)| \psi(x) r(t, x) \mathrm{d} x \\
& \leq \frac{C}{a}(t+1)^{3 \sqrt{N} /\left(2 \lambda_{*}\right)} e^{-\sqrt{N} a}\left[\left(t-t_{*}+1\right)^{-1 / 2}+e^{-c\left(t-t_{*}\right)^{2} / a}\right] .
\end{aligned}
$$

Taking into account (5.10), we see that (5.1) follows. 


\section{The late times}

We finish with the times $t \geq a$. In this regime, $p$ and $q_{\mathrm{e}}$ should be exponentially negligible. However, since this time period is unbounded, we must take care to ensure that $\dot{I}(t) / I(t)$ is integrable in time, and, in fact, small. We will prove the following lemma.

Lemma 6.1 There exists $C>0$ such that for all $t \geq a$ we have

$$
\frac{|\dot{I}(t)|}{I(t)} \leq \frac{C}{t^{3 / 2}}
$$

After integration, Lemma 6.1 implies that

$$
\log \frac{I(\infty)}{I(a)}=O\left(a^{-1 / 2}\right)
$$

Note that the lower bound (5.10) still holds, so it suffices to show that

$$
|\dot{I}(t)| \leq \frac{C}{t^{3 / 2}} a^{3 \sqrt{N} /\left(2 \lambda_{*}\right)} e^{-\sqrt{N} a} \quad \text { for } t \geq a
$$

\section{The free contribution}

It is simple to control the free contribution at late times, since it has decayed into irrelevance.

Lemma 6.2 There exist $c>0$ and $C>0$ such that for all $t \geq a$,

$$
\int_{\mathbb{R}}\left[\psi(x)+\psi^{\prime}(x)+|E(t, x)| \psi(x)\right] p(t, x) \mathrm{d} x \leq C e^{-(\sqrt{N}+c) a} e^{-c t} .
$$

Proof. For $x \leq 0$ we recall that $E$ is bounded and we simply use (5.4) and (3.12), which give

$$
\int_{\mathbb{R}_{-}}\left[\psi(x)+\psi^{\prime}(x)+|E(t, x)| \psi(x)\right] p(t, x) \mathrm{d} x \leq C \sqrt{t} \Lambda(t ; a) \leq C t^{C} e^{-a \theta(t / a)}
$$

for $\theta$ defined in (4.29). Now, $\min \theta=\sqrt{N}$ is uniquely attained at $\xi_{*}=1 /(2 \sqrt{N})<1$, and $\theta(\xi) \geq N \xi$. If $c>0$ is sufficiently small, it follows that

$$
\theta(\xi) \geq \sqrt{N}+c+c \xi \text { for all } \xi \geq 1 .
$$

Together with (6.4), this implies

$$
\int_{\mathbb{R}_{-}}\left[\psi(x)+\psi^{\prime}(x)+|E(t, x)| \psi(x)\right] p(t, x) d x \leq C e^{-(\sqrt{N}+c) a} e^{-c t} .
$$

For $x \geq 0$, recall that $\psi(x) \leq C(1+x)$ and $\psi^{\prime}(x) \leq C$. Then (3.11) implies

$$
\int_{\mathbb{R}_{+}}\left[\psi(x)+\psi^{\prime}(x)+|E(t, x)| \psi(x)\right] p(t, x) \mathrm{d} x \leq C \Lambda(t ; a) \int_{0}^{\infty}(1+x) e^{-a x /(2 t)} \mathrm{d} x \leq C t^{C} e^{-a \theta(t / a)} .
$$

Again, (6.5) yields

$$
\int_{\mathbb{R}_{+}}\left[\psi(x)+\psi^{\prime}(x)+|E(t, x)| \psi(x)\right] p(t, x) d x \leq C e^{-(\sqrt{N}+c) a} e^{-c t} .
$$

Combining this with (6.6), we obtain (6.3). 


\section{The main corrector contribution}

Next, we control $q_{\mathrm{m}}$ at late times.

Lemma 6.3 There exist $c>0$ and $C>0$ such that for all $t \geq a$,

$$
\int_{\mathbb{R}} \psi^{\prime}(x) q_{\mathrm{m}}(t, x) \mathrm{d} x \leq \frac{C}{\sqrt{t}} a^{3 \sqrt{N} /\left(2 \lambda_{*}\right)} e^{-\sqrt{N} a}
$$

and

$$
\int_{\mathbb{R}}|E(t, x)| \psi(x) q_{\mathrm{m}}(t, x) \mathrm{d} x \leq \frac{C}{t^{2}} a^{3 \sqrt{N} /\left(2 \lambda_{*}\right)} e^{-\sqrt{N} a} .
$$

Proof. We recall the Duhamel formula (4.22) for $q^{s}$, the solution to (4.23). We also recall Lemma 4.4: for $t \geq s \geq t_{-}$,

$$
\begin{aligned}
& \int_{\mathbb{R}} \psi^{\prime}(x) q^{s}(t, x) \mathrm{d} x \leq C \frac{s^{2}}{a^{2}} \Lambda(s ; a)(t-s+1)^{-1 / 2}, \\
& \int_{\mathbb{R}}|E(t, x)| \psi(x) q^{s}(t, x) \mathrm{d} x \leq C \frac{s^{2}}{a^{2}} \Lambda(s ; a) t^{-1 / 2}(t-s+1)^{-3 / 2} .
\end{aligned}
$$

To bound the contributions of $q_{\mathrm{m}}$, we integrate over $s \in\left[t_{-}, t\right]$. We rely on two different estimates for $\Lambda$. When $s \in\left[t_{-}, a\right]$, (4.32) implies:

$$
\frac{s^{2}}{a^{2}} \Lambda(s ; a) \leq \frac{C}{\sqrt{a}} a^{3 \sqrt{N} /\left(2 \lambda_{*}\right)} e^{-\sqrt{N} a} e^{-\frac{c\left(s-t_{*}\right)^{2}}{a}} \quad \text { for } s \in\left[t_{-}, a\right] .
$$

This estimate cannot hold for $s \geq a$, since $\theta$ is not uniformly convex. Nonetheless, (6.5) yields:

$$
\frac{s^{2}}{a^{2}} \Lambda(s ; a) \leq C s^{2} a^{C} e^{-a \theta(a / s)} \leq C e^{-(\sqrt{N}+c) a} e^{-c s} \text { for } s \in[a, \infty) .
$$

We first use (6.9) and (6.10) to control the contributions of $q^{s}$ when $s \in\left[t_{-}, a\right]$. Recalling the definition (5.13) of $Z_{\alpha}(t)$ and noticing from (5.14) that $Z_{1 / 2}(t) \leq C \sqrt{a / t}$ for $t \geq a$, we find

$$
\begin{aligned}
\int_{t_{-}}^{a} \int_{\mathbb{R}} \psi^{\prime}(x) q^{s}(t, x) \mathrm{d} x \mathrm{~d} s & \leq \frac{C}{\sqrt{a}} a^{3 \sqrt{N} /\left(2 \lambda_{*}\right)} e^{-\sqrt{N} a} \int_{t_{-}}^{a}(t-s+1)^{-1 / 2} e^{-c\left(s-t_{*}\right)^{2} / a} \mathrm{~d} s \\
& \leq \frac{C}{\sqrt{t}} a^{3 \sqrt{N} /\left(2 \lambda_{*}\right)} e^{-\sqrt{N} a} .
\end{aligned}
$$

Similarly, noticing from $(5.14)$ that $Z_{3 / 2}(t) \leq C \sqrt{a} / t^{3 / 2}$, we obtain

$$
\begin{aligned}
\int_{t_{-}}^{a} \int_{\mathbb{R}}|E(t, x)| \psi(x) q^{s}(t, x) \mathrm{d} x \mathrm{~d} s & \leq \frac{C}{\sqrt{a t}} a^{3 \sqrt{N} /\left(2 \lambda_{*}\right)} e^{-\sqrt{N} a} \int_{t_{-}}^{a}(t-s+1)^{-3 / 2} e^{-c\left(s-t_{*}\right)^{2} / a} \mathrm{~d} s \\
& \leq \frac{C}{t^{2}} a^{3 \sqrt{N} /\left(2 \lambda_{*}\right)} e^{-\sqrt{N} a} .
\end{aligned}
$$

Next, we use (6.9) and (6.11) to control the contributions of $q^{s}$ when $s \geq a$. To do so, we rely on the following bound: for each $\alpha \geq 0$ and $t>0$,

$$
\int_{0}^{t} e^{-c s}(t-s+1)^{-\alpha} \mathrm{d} s \leq \int_{0}^{t / 2} e^{-c s}\left(\frac{t}{2}+1\right)^{-\alpha} \mathrm{d} s+\int_{t / 2}^{t} e^{-c s} \mathrm{~d} s \leq C_{\alpha}(t+1)^{-\alpha} .
$$

Thus

$$
\int_{a}^{t} \int_{\mathbb{R}} \psi^{\prime}(x) q^{s}(t, x) \mathrm{d} x \mathrm{~d} s \leq C e^{-(\sqrt{N}+c) a} \int_{a}^{t}(t-s+1)^{-1 / 2} e^{-c s} \mathrm{~d} s \leq \frac{C}{\sqrt{t}} e^{-(\sqrt{N}+c) a}
$$


and

$$
\int_{a}^{t} \int_{\mathbb{R}}|E(t, x)| \psi(x) q^{s}(t, x) \mathrm{d} x \mathrm{~d} s \leq \frac{C}{\sqrt{t}} e^{-(\sqrt{N}+c) a} \int_{a}^{t}(t-s+1)^{-3 / 2} e^{-c s} \mathrm{~d} s \leq \frac{C}{t^{2}} e^{-(\sqrt{N}+c) a} .
$$

We recall that

$$
\begin{aligned}
& \int_{\mathbb{R}} \psi^{\prime}(x) q_{\mathrm{m}}(t, x) \mathrm{d} x=\int_{t_{-}}^{t} \int_{\mathbb{R}} \psi^{\prime}(x) q^{s}(t, x) \mathrm{d} x \mathrm{~d} s, \\
& \int_{\mathbb{R}}|E(t, x)| \psi(x) q_{\mathrm{m}}(t, x) \mathrm{d} x=\int_{t_{-}}^{t} \int_{\mathbb{R}}|E(t, x)| \psi(x) q^{s}(t, x) \mathrm{d} x \mathrm{~d} s .
\end{aligned}
$$

Therefore (6.12) and (6.15) imply (6.7), while (6.13) and (6.16) imply (6.8).

\section{The proof of Lemma 6.1}

First, we recall (5.10):

$$
I(t) \geq c a^{3 \sqrt{N} /\left(2 \lambda_{*}\right)} e^{-\sqrt{N} a} \text { for } t \geq t_{*} .
$$

Next, collecting Lemmas 3.2, 6.2, and 6.3, we see that (2.35) gives

$$
|\dot{I}(t)| \leq \frac{3}{2 \lambda_{*}(t+1)} \int_{\mathbb{R}} \psi^{\prime}(x) r(t, x) \mathrm{d} x+\int_{\mathbb{R}}|E(t, x)| r(t, x) \psi(x) \mathrm{d} x \leq \frac{C}{t^{3 / 2}} a^{3 \sqrt{N} /\left(2 \lambda_{*}\right)} e^{-\sqrt{N} a} .
$$

We obtain (6.1) from (6.17) and (6.18).

\section{Proof of the main result}

As we have mentioned, Proposition 2.4 is an immediate consequence of Lemmas 4.1, 5.1, and 6.1. Combining (4.2), (5.2) and (6.2), we find

$$
\log \frac{I(\infty)}{I(0)}=\frac{3 \sqrt{N}}{2 \lambda_{*}} \log \frac{a}{2 \sqrt{N}}+O\left(a^{-1 / 2}\right) .
$$

Exponentiating, we obtain Proposition 2.4.

\section{The early corrector — Proof of Lemma 3.2}

Given $\varepsilon_{0} \in(0,1)$, to be chosen later, Lemma 3.1 ensures the existence of $K \geq 0$ such that

$$
N\left(1-\varepsilon_{0}\right) \leq V(t, x) \text { for all } t>0 \text { and } x<-K .
$$

The same lemma implies that

$$
N-V(t, x) \leq B e^{\gamma_{*} x}
$$

Hence if $\tilde{q}$ solves

$$
\partial_{t} \tilde{q}=\partial_{x}^{2} \tilde{q}-\frac{3}{2 \lambda_{*}(t+1)} \partial_{x} \tilde{q}-N\left(1-\varepsilon_{0}\right) \mathbb{1}_{(-\infty,-K)}(x) \tilde{q}+B e^{\gamma_{*} x} \mathbb{1}_{\left[0, t_{-}\right]}(t) p, \quad \tilde{q}(0, x)=0,
$$

comparison with (3.14) implies

$$
q_{\mathrm{e}}(t, x) \leq \tilde{q}(t, x) .
$$


To estimate $\tilde{q}$, we decompose it into two parts, $\rho$ and $\sigma$

$$
q_{\mathrm{e}}(t, x) \leq \tilde{q}(t, x)=\rho(t, x)+\sigma(t, x),
$$

where $\rho$ is the solution to

$$
\partial_{t} \rho=\partial_{x}^{2} \rho-\frac{3}{2 \lambda_{*}(t+1)} \partial_{x} \rho-N\left(1-\varepsilon_{0}\right) \rho+B e^{\gamma_{*} x} \mathbb{1}_{\left[0, t_{-}\right]}(t) p, \quad \rho(0, x)=0,
$$

and $\sigma$ the solution to

$$
\partial_{t} \sigma=\partial_{x}^{2} \sigma-\frac{3}{2 \lambda_{*}(t+1)} \partial_{x} \sigma-N\left(1-\varepsilon_{0}\right) \mathbb{1}_{(-\infty,-K)}(x) \sigma+N\left(1-\varepsilon_{0}\right) \mathbb{1}_{[-K, \infty)}(x) \rho, \quad \sigma(0, x)=0 .
$$

We will be able to estimate $\rho$ more or less explicitly. For $\sigma$, we emphasize that the forcing in the right side of (7.2) is supported on $[-K, \infty)$. That is, unlike $r$ and $q_{\mathrm{e}}, \sigma$ is never forced deep in $\mathbb{R}_{-}$. There is thus no need to estimate it with a further corrector-we can control it with Lemma 3.3.

Lemma 3.2 is a consequence of the two following lemmas:

Lemma 7.1 There exist $C, c>0$ independent of $a$ and $t$ such that for all $a \geq 1$ and $t \geq 0$ :

$$
\int_{\mathbb{R}} \psi(x) \rho(t, x) \mathrm{d} x+\int_{\mathbb{R}} \psi^{\prime}(x) \rho(t, x) \mathrm{d} x+\int_{\mathbb{R}} \psi(x)|E(t, x)| \rho(t, x) \mathrm{d} x \leq C e^{-(\sqrt{N}+c) a-c t}
$$

Lemma 7.2 There exist $C, c>0$ independent of $a$ and $t$ such that for all $a \geq 1$ and $t \geq 0$ :

$$
\begin{aligned}
& \int_{\mathbb{R}} \psi(x) \sigma(t, x) \mathrm{d} x \leq C e^{-(\sqrt{N}+c) a}, \\
& \int_{\mathbb{R}} \psi^{\prime}(x) \sigma(t, x) \mathrm{d} x \leq \frac{C}{\sqrt{t+1}} e^{-(\sqrt{N}+c) a}, \\
& \int_{\mathbb{R}} \psi(x)|E(t, x)| \sigma(t, x) \mathrm{d} x \leq \frac{C}{(t+1)^{2}} e^{-(\sqrt{N}+c) a} .
\end{aligned}
$$

\section{Proof of Lemma 7.1}

We use the Duhamel formula to write the solution to (7.1) as

$$
\rho(t, x)=\int_{0}^{t \wedge t_{-}} \rho^{s}(t, x) \mathrm{d} s .
$$

Here, $\rho^{s}(t, x)$ is the solution to

$$
\partial_{t} \rho^{s}=\partial_{x}^{2} \rho^{s}-\frac{3}{2 \lambda_{*}(t+1)} \partial_{x} \rho^{s}-N\left(1-\varepsilon_{0}\right) \rho^{s} \text { for } t>s, \quad \rho^{s}(s, x)=B e^{\gamma_{*} x} p(s, x) .
$$

Recall the expression (3.2) of $p(t, x)$ and the definition (4.8) of $\mu(t)$ :

$$
\mu(t)=-a+\frac{3}{2 \lambda_{*}} \log (t+1) .
$$

This allows us to write the initial condition $\rho^{s}(s, x)$ as a Gaussian:

$$
\begin{aligned}
\rho^{s}(s, x)=B e^{\gamma_{*} x} p(s, x) & =B e^{\gamma_{*} x} \frac{1}{\sqrt{4 \pi s}} \exp \left\{-N s-\frac{1}{4 s}[x-\mu(s)]^{2}\right\} \\
& =\frac{B}{\sqrt{4 \pi s}} e^{\gamma_{*}\left(\mu(s)+\gamma_{*} s\right)} \exp \left\{-N s-\frac{1}{4 s}\left[x-\mu(s)-2 \gamma_{*} s\right]^{2}\right\} .
\end{aligned}
$$


One can then verify that

$$
\rho^{s}(t, x)=\frac{B}{\sqrt{4 \pi t}} e^{-N \varepsilon_{0} s} e^{-N\left(1-\varepsilon_{0}\right) t} e^{\gamma_{*}\left(\mu(s)+\gamma_{*} s\right)} \exp \left[-\frac{\left(x-\mu(t)-2 \gamma_{*} s\right)^{2}}{4 t}\right]
$$

satisfies both the initial condition and (7.5). The Duhamel formula (7.4) implies

$$
\int_{\mathbb{R}} \psi(x) \rho(t, x) \mathrm{d} x=\int_{0}^{t \wedge t_{-}} \int_{\mathbb{R}} \psi(x) \rho^{s}(t, x) \mathrm{d} x \mathrm{~d} s
$$

for all $t \geq 0$.

To bound the first term in (7.3), it suffices to show that for all $t \geq 0$ and $s \leq t \wedge t_{-}$,

$$
\int_{\mathbb{R}} \psi(x) \rho^{s}(t, x) \mathrm{d} x \leq C(1+t)^{C} e^{-(\sqrt{N}+c) a-c t}
$$

for some $c>0, C>0$. Then, the integral over $s \leq t_{-} \leq a$ in (7.7) gives at most a factor $a$ which can be absorbed, together with the $(1+t)^{C}$ factor, into in the exponential decay by making $c$ smaller.

We now show (7.8). It follows from (2.30) that $\psi(x) \leq C e^{\sqrt{N} x}$ for some $C$; then, in (7.6), we obtain

$$
\psi(x) \rho^{s}(t, x) \leq e^{\sqrt{N} x} \rho^{s}(t, x) \leq \frac{C}{\sqrt{t}} e^{-N\left(1-\varepsilon_{0}\right) t} e^{\gamma_{*}\left(\mu(s)+\gamma_{*} s\right)} e^{\sqrt{N} x} \exp \left[-\frac{\left(x-\mu(t)-2 \gamma_{*} s\right)^{2}}{4 t}\right] .
$$

Now,

$$
e^{\sqrt{N} x} \exp \left[-\frac{\left(x-\mu(t)-2 \gamma_{*} s\right)^{2}}{4 t}\right]=e^{\sqrt{N}\left(\mu(t)+2 \gamma_{*} s+\sqrt{N} t\right)} \exp \left[-\frac{\left(x-\mu(t)-2 \gamma_{*} s-2 \sqrt{N} t\right)^{2}}{4 t}\right] .
$$

Hence (7.9) yields

$$
\begin{aligned}
\int_{\mathbb{R}} \psi(x) \rho^{s}(t, x) \mathrm{d} x & \leq C \exp \left\{-N\left(1-\varepsilon_{0}\right) t+\gamma_{*}\left(\mu(s)+\gamma_{*} s\right)+\sqrt{N}\left(\mu(t)+2 \gamma_{*} s+\sqrt{N} t\right)\right\} \\
& =C \exp \left\{\sqrt{N} \mu(t)+N \varepsilon_{0} t+\gamma_{*} \mu(s)+\gamma_{*}\left(\gamma_{*}+2 \sqrt{N}\right) s\right\} \\
& \leq C(1+t)^{C} \exp \left\{N \varepsilon_{0} t-\left(\sqrt{N}+\gamma_{*}\right) a+\gamma_{*}\left(\gamma_{*}+2 \sqrt{N}\right) s\right\} .
\end{aligned}
$$

(In the last line, we replaced $\mu(s)$ and $\mu(t)$ by their expression (4.8), and then used $s \leq t$ for the logarithmic terms.) Pick $c>0$, and then $\varepsilon_{0}$ such that $N \varepsilon_{0} \leq c$. We see that (7.8) holds if

$$
c t-\gamma_{*} a+\gamma_{*}\left(\gamma_{*}+2 \sqrt{N}\right) s \leq-c a-c t .
$$

We now consider, till the end of this proof, the case where (7.12) does not hold, i.e. the case where

$$
\gamma_{*}\left(\gamma_{*}+2 \sqrt{N}\right) s>\left(\gamma_{*}-c\right) a-2 c t .
$$

In that case, the bound $\psi(c) \leq C e^{\sqrt{N} x}$ that we used to derive (7.11) is not good enough for $x>0$. We now show that if $c>0$ is small enough, the centering term in (7.10) satisfies

$$
\mu(t)+2 \gamma_{*} s+2 \sqrt{N} t>c a .
$$

Indeed, recall that $\mu(t)>-a$ and $\sqrt{N}>1$. If $t>a$, then (7.14) is obvious. If $t<a$, then (7.13) and $t>s$ imply that

$$
\mu(t)+2 \gamma_{*} s+2 \sqrt{N} t \geq-a+2\left(\gamma_{*}+\sqrt{N}\right) s \geq-a+\left[\frac{2\left(\gamma_{*}+\sqrt{N}\right)}{\gamma_{*}+2 \sqrt{N}}\right] \frac{\gamma_{*}-3 c}{\gamma_{*}} a
$$


As the factor in square brackets is strictly larger than 1, it is possible to choose $c>0$ small enough independent of $a$ such that (7.14) holds. Thus when $x<0$, (7.13) implies

$$
\exp \left[-\frac{\left(x-\mu(t)-2 \gamma_{*} s-2 \sqrt{N} t\right)^{2}}{4 t}\right] \leq \exp \left[-\frac{\left(\mu(t)+2 \gamma_{*} s+2 \sqrt{N} t\right)^{2}}{4 t}+\frac{\operatorname{cax}}{2 t}\right] .
$$

Recalling (7.6), (7.9), and (7.10), we obtain

$$
e^{\sqrt{N} x} \rho^{s}(t, x) \leq C \rho^{s}(t, 0) \exp \left(\frac{c a x}{2 t}\right) \quad \text { for } x<0 .
$$

Therefore,

$$
\int_{-\infty}^{0} \psi(x) \rho^{s}(t, x) \mathrm{d} x \leq C \frac{t}{a} \rho^{s}(t, 0) .
$$

We now evaluate the integral over $x>0$, still assuming (7.13). In this region, we use the bound $\psi(x) \leq C(1+x)$. In the expression (7.6) of $\rho^{s}(t, x)$, we start by getting rid of the logarithmic terms that appear in $\mu(t)$ and $\mu(s)$. We employ (4.25) with some $\varepsilon$ to be chosen later. Recalling that $N \varepsilon_{0} \leq c,(7.6)$ implies:

$$
\rho^{s}(t, x) \leq \tilde{\rho}^{s}(t, x) \quad \text { with } \quad \tilde{\rho}^{s}(t, x)=\frac{C}{\sqrt{t}} \exp \left[2 c t-N t-\gamma_{*}\left(a-\gamma_{*} s\right)-(1-\varepsilon) \frac{\left(x+a-2 \gamma_{*} s\right)^{2}}{4 t}\right] .
$$

The term $2 c t$ has two contributions. On the one hand, $N \varepsilon_{0} t \leq c t$. On the other, the logarithmic term in $\mu(s)$ is sublinear: $2 \gamma_{*} /\left(3 \lambda_{*}\right) \log (1+s) \leq c t+C$.

Since $s \leq t_{-} \leq t_{*}=a /(2 \sqrt{N})$ and $\gamma_{*} \leq \sqrt{N}$, we have $a-2 \gamma_{*} s \geq 0$ and

$$
\tilde{\rho}^{s}(t, x) \leq \tilde{\rho}^{s}(t, 0) \exp \left[-(1-\varepsilon) \frac{x^{2}}{4 t}\right] \quad \text { for } x>0 .
$$

Then

$$
\int_{0}^{\infty} \psi(x) \rho^{s}(t, x) \mathrm{d} x \leq C \tilde{\rho}^{s}(t, 0) \int_{0}^{\infty}(1+x) \exp \left[-(1-\varepsilon) \frac{x^{2}}{4 t}\right] \mathrm{d} x \leq C(\sqrt{t}+t) \tilde{\rho}^{s}(t, 0) .
$$

Combining (7.15) and (7.17), we have shown that, if (7.13) holds, we have

$$
\int_{\mathbb{R}} \psi(x) \rho^{s}(t, x) \mathrm{d} x \leq C(\sqrt{t}+t) \tilde{\rho}^{s}(t, 0) .
$$

We now evaluate $\tilde{\rho}^{s}(t, 0)$ and show that if $\epsilon$ is chosen small enough, then there exists $C>0$ and $c>0$ such that, for all $t>0$ and all $s \leq t \wedge t_{-}$,

$$
\tilde{\rho}^{s}(t, 0) \leq \frac{C}{\sqrt{t}} \exp [-(\sqrt{N}+c) a-c t]
$$

This will imply (7.8), and then Lemma 7.1 for the term with $\psi$. We have from (7.16)

$$
\begin{aligned}
\tilde{\rho}^{s}(t, 0) & =\frac{C}{\sqrt{t}} \exp \left[2 c t-N t-\gamma_{*}\left(a-\gamma_{*} s\right)-(1-\varepsilon) \frac{\left(a-2 \gamma_{*} s\right)^{2}}{4 t}\right] \\
& =\frac{C}{\sqrt{t}} \exp \left[2 c t-N t-\gamma_{*}\left(a-\gamma_{*} s\right)-(1-\varepsilon)\left(\frac{a^{2}}{4 t}-\frac{\gamma_{*} s}{t}\left(a-\gamma_{*} s\right)\right)\right] \\
& \leq \frac{C}{\sqrt{t}} \exp \left[2 c t-(1-\varepsilon)\left(N \frac{t}{a}+\frac{a}{4 t}\right) a-\gamma_{*}\left(a-\gamma_{*} s\right)\left(1-(1-\varepsilon) \frac{s}{t}\right)\right] \\
& \leq \frac{C}{\sqrt{t}} \exp \left[2 c t-(1-\varepsilon) \theta\left(\frac{t}{a}\right) a-\gamma_{*}\left(a-\gamma_{*} s\right)\left(1-\frac{s}{t}\right)\right]
\end{aligned}
$$


with $\theta(\xi):=N \xi+1 /(4 \xi)$. Here we have used $a-\gamma_{*} s>0$. Notice that $\theta(\xi)$ reaches its minimum at $\xi_{*}=t_{*} / a=1 /(2 \sqrt{N})$ with $\theta\left(\xi_{*}\right)=\sqrt{N}$. Let $t_{0}=\left(t_{-}+t_{*}\right) / 2$. Notice also that $t_{0} / a$ does not depend on $a$ and that $t_{0} / a<\xi_{*}$. We choose $c$ small enough that $\theta\left(t_{0} / a\right)>\sqrt{N}+5 c$, and then $\varepsilon$ small enough that $(1-\varepsilon) \theta\left(t_{0} / a\right)>\sqrt{N}+4 c$. Then, for $t \leq t_{0}$, we have $\theta(t / a) \geq \theta\left(t_{0} / a\right)$ and

$$
\tilde{\rho}^{s}(t, 0) \leq \frac{C}{\sqrt{t}} \exp \left[2 c t-(1-\varepsilon) \theta\left(t_{0} / a\right) a\right] \leq \frac{C}{\sqrt{t}} \exp [2 c t-(\sqrt{N}+4 c) a],
$$

where we used $s \leq t$ and $s \leq t_{-} \leq t_{*}=a /(2 \sqrt{N}) \leq a / \gamma_{*}$. Since $t \leq t_{0} \leq a$, this implies (7.18).

We now consider $t \in\left[t_{0}, a\right]$. Using $\theta(t / a) \geq \theta\left(\xi_{*}\right)=\sqrt{N}$ and $s \leq t_{-}=\xi_{-} a$,

$$
\tilde{\rho}^{s}(t, 0) \leq \frac{C}{\sqrt{t}} \exp \left[2 c t-(1-\varepsilon) \sqrt{N} a-a \gamma_{*}\left(1-\gamma_{*} \xi_{-}\right)\left(1-\frac{t_{-}}{t_{0}}\right)\right] .
$$

Notice that $t_{-} / t_{0}$ is independent of $a$ and strictly smaller than one. We make $c$ small enough that $\gamma_{*}\left(1-\gamma_{*} \xi_{-}\right)\left(1-t_{-} / t_{0}\right)>5 c$ and then $\varepsilon$ small enough that $(1-\varepsilon) \sqrt{N} \geq \sqrt{N}-c$. Then, since $t \leq a$, we obtain again the bound (7.18).

Finally, we consider $t \geq a$. As $\theta$ is convex, we have for $\xi \geq 1$

$$
\theta(\xi) \geq \theta(1)+\theta^{\prime}(1)(\xi-1)=\frac{1}{2}+\left(N-\frac{1}{4}\right) \xi \geq \sqrt{N}+\frac{1}{4}+(N-\sqrt{N}) \xi
$$

and

$$
\tilde{\rho}^{s}(t, 0) \leq \frac{C}{\sqrt{t}} \exp \left[2 c t-(1-\varepsilon)\left(\sqrt{N}+\frac{1}{4}\right) a-(1-\varepsilon)(N-\sqrt{N}) t\right] .
$$

It is then clear that by taking $c$ and $\varepsilon$ small enough, we have again (7.18).

This concludes the proof that the first term in (7.3) is bounded as stated by Lemma 7.1. In this proof, we only used that $\psi(x) \leq C \exp (\sqrt{N} x)$ for $x<0$ and $\psi(x) \leq C(1+x)$ for $x \geq 0$. Since $\psi^{\prime}(x)$ satisfies the same bound and $E$ is bounded, the other terms in (7.3) are bounded in the same way as the first term and the proof of Lemma 7.1 is complete.

\section{Proof of Lemma 7.2}

We now turn to the solution $\sigma$ of (7.2):

$$
\partial_{t} \sigma=\partial_{x}^{2} \sigma-\frac{3}{2 \lambda_{*}(t+1)} \partial_{x} \sigma-N\left(1-\varepsilon_{0}\right) \mathbb{1}_{(-\infty,-K)}(x) \sigma+N\left(1-\varepsilon_{0}\right) \mathbb{1}_{[-K, \infty)}(x) \rho, \quad \sigma(0, x)=0 .
$$

Let us start by showing that

$$
\int_{\mathbb{R}} \psi(x) \sigma(t, x) \mathrm{d} x=O\left(e^{-(\sqrt{N}+c) a}\right) .
$$

We again represent $\sigma(t, x)$ via the Duhamel formula:

$$
\sigma(t, x)=\int_{0}^{t} \sigma^{s}(t, x) \mathrm{d} s
$$

Here, $\sigma^{s}(t, x)$, defined for $0<s<t$, is the solution to

$$
\begin{aligned}
& \partial_{t} \sigma^{s}=\partial_{x}^{2} \sigma^{s}-\frac{3}{2 \lambda_{*}(t+1)} \partial_{x} \sigma^{s}-N\left(1-\varepsilon_{0}\right) \mathbb{1}_{(-\infty,-K)}(x) \sigma^{s}, \quad t>s, \\
& \sigma^{s}(s, x)=N\left(1-\varepsilon_{0}\right) \mathbb{1}_{[-K, \infty)}(x) \rho(s, x) .
\end{aligned}
$$

Our first step will be to bound the initial condition in (7.20) with the following Lemma. 
Lemma 7.3 There exist $C>0, c>0$, and $\kappa>0$ such that for all $s \geq 0$ and all $x \in \mathbb{R}$ :

$$
\sigma^{s}(s, x) \leq C \exp \left[-(\sqrt{N}+c) a-c s-\kappa \min \left\{1, \frac{a}{s}\right\} x_{+}-\frac{x^{2}}{8 s}\right] \mathbb{1}_{[-K, \infty)}(x) .
$$

with $x_{+}=\max \{x, 0\}$.

Proof. Recall from (7.20) that $\sigma^{s}(s, x)=C \mathbb{1}_{[-K, \infty)}(x) \rho(s, x)$ with $\rho(t, x)$ given by $(7.4)$ :

$$
\rho(t, x)=\int_{0}^{t \wedge t_{-}} \rho^{s}(t, x) \mathrm{d} s .
$$

We use $\rho^{s}(t, x) \leq \tilde{\rho}^{s}(t, x)$ with $\tilde{\rho}^{s}(t, x)$ given in (7.16). It suffices to show that there exists $\kappa>0$ such that, for all $s \leq t \wedge t_{-}$,

$$
\tilde{\rho}^{s}(t, x) \leq \frac{C}{\sqrt{t}} \exp \left[-(\sqrt{N}+c) a-c t-\kappa \min \left\{\left(1, \frac{a}{t}\right\} x_{+}-\frac{x^{2}}{8 t}\right] \quad \text { for } x>-K\right.
$$

Then $\rho(t, x) \leq C \sqrt{t} \exp [\ldots]$, and the $\sqrt{t}$ can be absorbed in the exponential by making $c$ smaller. We now show (7.22).

We first assume that $t \leq \delta a$, for some small $\delta>0$ to be chosen. For such small times, the Gaussian term in (7.16) controls everything. We use the following result: for any $\varepsilon \in[0,1 / 2)$, any $\kappa \geq 0$, any $\lambda \geq 0$ and any $K \geq 0$, there exists $\delta^{\prime}>0$ such that, if $b$ is large enough,

$$
(1-\varepsilon) \frac{(x+b)^{2}}{4 t} \geq \frac{x^{2}}{8 t}+\kappa x+\lambda b \quad \text { for all } t \leq \delta^{\prime} b \text { and } x \geq-K .
$$

Indeed, taking $\varepsilon=0$ for simplicity, (7.23) is equivalent to $x^{2}+2 \beta x+\gamma \geq 0$ with $\beta=2 b-4 \kappa t$ and $\gamma=2 b^{2}-8 \lambda b t$. By making $\delta^{\prime}$ small enough, we have $\beta \in[1.9 b, 2 b]$ and $\gamma \in\left[1.9 b^{2}, 2 b^{2}\right]$ for $t \leq \delta^{\prime} b$. Then, (7.23) holds for $x \geq-\beta+\sqrt{\beta^{2}-\gamma}$, where $-\beta+\sqrt{\beta^{2}-\gamma}<(-1.9+\sqrt{4-1.9}) b \approx-0.45 b$, which is smaller than $-K$ for $b$ large enough.

Then, in (7.16), we notice that $b:=a-2 \gamma_{*} s \geq a-2 \gamma_{*} t_{-}=a\left(1-2 \gamma_{*} \xi_{-}\right)$, with $1-2 \gamma_{*} \xi_{-}>0$. We use (7.23) with this $b$ and $\lambda=(\sqrt{N}+c) /\left(1-2 \gamma_{*} \xi_{-}\right)$to obtain (7.22) for $t \leq \delta^{\prime} b \leq \delta a$ with $\delta=\delta^{\prime}\left(1-2 \gamma_{*} \xi_{-}\right)$. (We wrote $2 c t-N t \leq-c t$ in (7.16) after assuming $c<N / 3$, and we used $\kappa x \geq \kappa x_{+}-\kappa K$ for $x \geq-K$.)

We now turn to $t \geq \delta a$. From (7.16) and (7.18), we have

$$
\begin{aligned}
\tilde{\rho}^{s}(t, x) & =\tilde{\rho}^{s}(t, 0) \exp \left[-(1-\varepsilon) \frac{x^{2}}{4 t}-(1-\varepsilon) \frac{a-2 \gamma_{*} s}{2 t} x\right] \\
& \leq \frac{C}{\sqrt{t}} \exp \left[-(\sqrt{N}+c) a-c t-\frac{x^{2}}{8 t}-(1-\varepsilon) \frac{a-2 \gamma_{*} s}{2 t} x\right]
\end{aligned}
$$

Pick $\kappa=(1-\varepsilon)\left(1-2 \gamma_{*} \xi_{-}\right) / 2$. Since $s \in\left[0, \xi_{-} a\right]$ and $t \geq \delta a$, we have

$$
(1-\varepsilon) \frac{a-2 \gamma_{*} s}{2 t} \in\left[\frac{\kappa a}{t}, \frac{1}{2 \delta}\right] \text {. }
$$

This implies that

$$
(1-\varepsilon) \frac{a-2 \gamma_{*} s}{2 t} x \geq \frac{\kappa a}{t} x_{+}-\frac{K}{2 \delta} \geq \kappa \min \left\{1, \frac{a}{t}\right\} x_{+}-\frac{K}{2 \delta} \quad \text { for all } x \geq-K .
$$


Using this bound in (7.24) concludes the proof of (7.22) and of Lemma 7.3.

The solution to (7.20) with the initial condition (7.21) can be treated by Lemma 3.3, with $\kappa_{-}=0$ and $\kappa_{+}=\kappa \min \{1, a / s\}$. For instance,

$$
\int_{\mathbb{R}} \psi(x) \sigma^{s}(t, x) \mathrm{d} x \leq C e^{-(\sqrt{N}+c) a-c s} \max \left\{\left(\frac{s}{a}\right)^{2}, 1\right\} .
$$

Since $a \geq 1$, we have $\max \left\{(s / a)^{2}, 1\right\} \leq C(\varepsilon) e^{\varepsilon s}$ for any $\varepsilon>0$. Thus, we can absorb $\max \left\{(s / a)^{2}, 1\right\}$ into the exponential factor $e^{-c s}$ by reducing $c$. After this operation, Lemma 3.3 yields:

$$
\begin{aligned}
& \int_{\mathbb{R}} \psi(x) \sigma^{s}(t, x) \mathrm{d} x \leq C e^{-(\sqrt{N}+c) a-c s}, \\
& \int_{\mathbb{R}} \psi^{\prime}(x) \sigma^{s}(t, x) \mathrm{d} x \leq C e^{-(\sqrt{N}+c) a-c s}(t-s+1)^{-1 / 2}, \\
& \int_{\mathbb{R}}|E(t, x)| \psi(x) \sigma^{s}(t, x) \mathrm{d} x \leq C e^{-(\sqrt{N}+c) a-c s}(t+1)^{-1 / 2}(t-s+1)^{-3 / 2} .
\end{aligned}
$$

In light of the Duhamel formula (7.19), we must integrate (7.25) over $s \in[0, t]$. Taking $\alpha \in\{1 / 2,3 / 2\}$ in (6.14), (7.19) and (7.25) imply Lemma 7.2.

\section{A The proof of Lemma 3.3}

To prove Lemma 3.3, we argue that the absorption on $(-\infty,-K)$ in $(3.16)$ acts similarly to the Dirichlet boundary condition.

We begin by constructing a family of supersolutions based on the Dirichlet problem. Recall that we defined

$$
\varphi(\lambda, x)=\frac{x}{\lambda^{3 / 2}} \exp \left(-\frac{x^{2}}{4 \lambda}\right) .
$$

Now let $v(t, x ; s)$, with $s \geq 0$, be the solution to

$$
\begin{array}{ll}
\partial_{t} v=\partial_{x}^{2} v-\frac{3}{2(t+s+1)} \partial_{x} v, & \text { for } t>0 \text { and } x>0, \\
v(t, 0)=0, & \text { for } t>0, \\
v(0, x)=\varphi(\lambda, x) & \text { for } x>0 .
\end{array}
$$

We define the " $\lambda$-adapted" self-similar variables

$$
\tau=\log \left(\frac{t+\lambda}{t}\right) \quad \text { and } \quad \eta=\frac{x}{\sqrt{t+\lambda}} .
$$

In these coordinates, $v$ satisfies

$$
\partial_{\tau} v=\partial_{\eta}^{2} v+\frac{\eta}{2} \partial_{\eta} v-m(\tau) \partial_{\eta} v, \quad v(0, \eta)=\varphi(1, \eta)
$$

with a drift

$$
m(\tau)=\frac{3}{2} \frac{\sqrt{\lambda} e^{\tau / 2}}{\lambda\left(e^{\tau}-1\right)+s+1} .
$$

We wish to argue that the drift term $m \partial_{\eta} v$ is negligible, so that $\varphi(\lambda+t, x)$ is an approximate solution to (A.1). To this end, note that if $s \geq c_{0} \lambda$ with $c_{0}>0$ fixed, we have

$$
m(\tau) \leq C s^{-1 / 2} \ll 1 .
$$

Combining Lemma 2.2 in [11] with methods from [17], we have the following analogue of Lemma 5.4. 
Lemma A.1 Fix $c_{0}>0$ and suppose that $s \geq c_{0} \lambda$. There exists $C\left(c_{0}\right)>0$ such that if $s \geq C$, then the solution $v(t, x ; s)$ to (A.1) has the form

$$
v(t, x)=\varphi(t+\lambda, x)+(t+\lambda)^{-3 / 2} v_{0}(t, x ; s),
$$

with

$$
\left\|\underline{v}_{0}(s)\right\|_{\mathcal{C}_{w}^{1}}+\left\|\underline{v}_{0}(s)\right\|_{L_{w}^{2}} \leq C s^{-1 / 2}
$$

for the weight $w=\exp \left[x^{2} /(6(t-s+1))\right]$.

This lemma also permits the construction of supersolutions for more complicated equations. If we have an absorbing potential on the left rather than a Dirichlet condition, we can join $v$ to a decaying exponential. We illustrate the construction with a simple example. Consider the equation

$$
\partial_{t} \nu=\partial_{x}^{2} \nu-\frac{3}{2(t+1+s)} \partial_{x} \nu-\mathbb{1}(x<2) \nu
$$

It is easy to check that

$$
A_{\lambda}(t, x)=\frac{1}{2}(t+\lambda)^{-3 / 2} \exp \left(\frac{x}{2}\right)
$$

is a supersolution to (A.2) on $(-\infty, 2]$, provided $\lambda \geq 2$. We can glue $A_{\lambda}(t, x)$ on the left to the solution to the Dirichlet problem (A.1) on the right, with no potential, to form a global supersolution to (A.2). For the hybrid to be a supersolution itself, we need its slope to decrease at the joint, which ensures that in a neighborhood of the joint, it is the minimum of two supersolutions, and thus a supersolution. We must thus take some care to achieve this.

To emphasize the dependence on $\lambda$, let $v_{\lambda}$ denote the solution to (A.1). If $\lambda \geq 2$, the graphs of $A_{\lambda}(0, x)$ and $\varphi_{\lambda}(x)$ intersect twice in [0,2]. By Lemma A.1, the same is true of $A_{\lambda}(t, x)$ and $v_{\lambda}(t, x ; s)$ for $s$ sufficiently large. Therefore, the hybrid function

$$
\Psi(t, x)= \begin{cases}A_{\lambda}(t, x) & \text { if } x \leq j(t) \\ v_{\lambda}(t, x ; s) & \text { if } x>j(t)\end{cases}
$$

where $j(t)$ is the rightmost point of intersection, is a supersolution to (A.2).

Now, consider $w$ as in Lemma 3.3 with $s \geq C$ fixed. Let $\bar{w}$ denote the solution to (3.16) and (3.17) with inequalities replaced by equalities. Then, by the comparison principle, we have $w \leq \bar{w}$, and it suffices to control $\bar{w}$. Next shift $\bar{w}$ so that it starts at time 0 . To bound $\bar{w}$ from above, we will piece together many supersolutions of a hybrid form.

In the following, we assume that $\lambda$ and $s$ satisfy the hypotheses of Lemma A.1 and $s \geq C^{\prime} \geq C$ for some $C^{\prime}$ to be determined. We say a function is hybrid if it is a spatial shift and constant multiple of the following form:

$$
\Phi(t, x)= \begin{cases}B(t+\lambda)^{-3 / 2} e^{-b x} & \text { for } x \leq j(t), \\ v_{\lambda}(t, x ; s) & \text { for } x>j(t) .\end{cases}
$$

Here $B, C^{\prime}>0, b<\alpha$, and $j:[0, \infty) \rightarrow \mathbb{R}_{+}$(uniformly bounded) are chosen so that $\Phi$ is continuous and $\partial_{x} \Phi$ decreases at the joint $x=j(t)$. If $\lambda$ is sufficiently large (depending on $b$ ), $\Phi$ is a supersolution to $(3.16)$. Let $\mathcal{H}$ denote the set of hybrids.

Notice that

$$
\int_{0}^{\infty} \varphi(\lambda, x) \mathrm{d} x \sim \lambda^{-\frac{1}{2}} \text { and } \int_{\mathbb{R}_{+}} x \varphi(\lambda, x) \mathrm{d} x \sim 1
$$


If $\Phi$ is a hybrid with parameter $\lambda$, Lemma A.1 ensures that it resembles $\varphi(t+\lambda, x)$ on $\mathbb{R}_{+}$, and it is straightforward to check that

$$
\begin{aligned}
& \int_{\mathbb{R}} \psi(x) \Phi(t, x) \mathrm{d} x \leq C, \\
& \int_{\mathbb{R}} \psi^{\prime}(x) \Phi(t, x) \mathrm{d} x \leq C \lambda^{-1 / 2}, \\
& \int_{\mathbb{R}} E(t, x) \psi(x) \Phi(t, x) \mathrm{d} x \leq C(t+1)^{-1 / 2}(t+\lambda)^{-3 / 2} .
\end{aligned}
$$

To control the evolution of $\bar{w}$, we use a sum of hybrid supersolutions to cover different spatial regions of the initial condition $\bar{w}(0, x)$. On $\mathbb{R}_{-}$, we choose $\Phi_{-} \in \mathcal{H}$ of the form

$$
\Phi_{-}(t, x)=B(t+\lambda)^{-3 / 2} e^{-b x}
$$

on the left, with some $b \in\left(\kappa_{-}, \alpha\right)$ and $\lambda \geq 1$. On the right, $\Phi_{-}$has the form $D v_{\lambda}$ for some $D>0$. Taking $B, D \gg 1$, we can ensure that $\bar{w}(0, x) \leq H_{-}(0, x)$ for $x \leq 1$.

We are thus left with the decaying tail of $\bar{w}(0, x)$ for $x \geq 1$. We cover this tail by a sequence of ever-wider Gaussians. Noting that $\|\varphi(\lambda, \cdot)\|_{\infty} \sim \lambda^{-1}$, we use the normalized functions $\lambda \varphi(\lambda, x)$ as building blocks. In particular, there exists universal $D_{+}>0$ such that

$$
\bar{w}(0, x) \leq \sum_{k=0}^{\log _{2} s+1} D_{+} \exp \left(-\kappa_{+} 2^{k}\right) 2^{k} \varphi\left(2^{k}, x\right) \quad \text { for all } x \geq 1 .
$$

Note that we are able to truncate the sum at $k=\log _{2} s+1$ because $\bar{w}(0, x)$ is bounded by the Gaussian $\exp \left\{-x^{2} /(8 s)\right\}$. Using (A.4), we construct hybrid supersolutions $\Phi_{k} \in \mathcal{H}$ equal to a shifted multiple of $v_{2^{k}}(t, x ; s)$ on the right. Note that the condition $k \leq \log _{2} s+1$ implies $\lambda=2^{k} \leq 2 s$. We thus satisfy the hypotheses of Lemma A.1.

By the comparison principle, we have

$$
\bar{w} \leq \Phi_{-}+\sum_{k=0}^{\log _{2} s+1} \Phi_{k} .
$$

The sum of the coefficients in (A.4) is bounded by $C \kappa_{+}^{-2}$. Therefore, (A.3) implies

$$
\begin{aligned}
& \int_{\mathbb{R}} \psi(x) \bar{w}(t, x) \mathrm{d} x \leq C \max \left\{\kappa_{+}^{2}, 1\right\}, \\
& \int_{\mathbb{R}} \psi^{\prime}(x) \bar{w}(t, x) \mathrm{d} x \leq C \max \left\{\kappa_{+}^{2}, 1\right\}(t+1)^{-1 / 2}, \\
& \int_{\mathbb{R}} e^{-\delta|x|} \psi(x) \bar{w}(t, x) \mathrm{d} x \leq C \max \left\{\kappa_{+}^{2}, 1\right\}(t+1)^{-3 / 2} .
\end{aligned}
$$

Now, (3.18)-(3.20) follow, after recalling the time change $w(t, x) \leq \bar{w}(t-s, x)$ due to our temporal shift of $\bar{w}$.

We have yet to handle $s \leq C^{\prime}$. We first note that $\Phi_{k}$ only depends on $\kappa_{+}$through a scalar multiple. Hence $C^{\prime}$ is independent of $\kappa_{+}$. When $s \leq C^{\prime}$, we can simply forget about the absorbing potential in (3.16), and allow our solution to evolve under a (drifting) heat equation until time $C^{\prime}$. This will certainly be a supersolution for (3.16). Since the solution at time $C^{\prime}$ will still be bounded by a Gaussian, we can run our argument above once we reach time $C^{\prime}$. This completes the proof of Lemma 3.3. 


\section{B The differentiability of the Bramson shift}

In this appendix, we justify (2.14).

Lemma B.1 The shift $s(y, a)$ is differentiable with respect to $y$. Moreover, for all $a>0$,

$$
\lim _{t \rightarrow \infty} z(t, x+m(t) ; a)=-\partial_{y} s(0, a) U^{\prime}\left(x-\bar{x}_{0}\right)
$$

uniformly in $x \in \mathbb{R}$.

Proof. We fix $a>0$ throughout the proof, and allow constants to implicitly depend on $a$. To establish differentiability, we prefer to work with a family of initial data defined on an open interval around $y=0$. We therefore define

$$
u(0, x ; y, a)=\mathbb{1}_{(-\infty, 0]}(x)+\mathbb{1}_{(-\infty, y-a]}(x)-\mathbb{1}_{(-\infty,-a]}(x) \quad \text { for } y \in \mathbb{R} .
$$

This coincides with the definition in $(2.8)$ when $y<0$, and satisfies

$$
\partial_{y} u(0, x ; y, a)=\delta(x-y+a)
$$

in the distributional sense for all $y \in \mathbb{R}$. We prove Lemma B.1 at $y=0$; the general case is similar. We are thus free to restrict our attention to $y \in(-1,1)$.

Again, we allow (B.2) to evolve under the Fisher-KPP equation (1.12) and obtain a solution $u(t, x ; y, a)$. We note that $u(0, x ; y, a)$ exceeds 1 on a compact interval when $y>0$, so we must extend the reaction $f$ beyond the interval $[0,1]$ to define the evolution. As mentioned earlier, standard parabolic estimates show that $z(t, x ; y, a)=\partial_{y} u(t, x ; y, a)$ exists and solves

$$
\partial_{t} z(t, x ; y, a)=\partial_{x}^{2} z(t, x ; y, a)+f^{\prime}(u(t, x ; y, a)) z(t, x ; y, a), \quad z(0, x ; y, a)=\delta(x-y+a) .
$$

Also, there exists $s(y, a) \in \mathbb{R}$ such that

$$
u(t, x+m(t) ; y, a) \rightarrow U(x-s(y, a)) \quad \text { as } t \rightarrow \infty
$$

uniformly in $x \in \mathbb{R}$. When $y=0$, Proposition 2.1 shows that $z$ converges to a multiple of $U_{0}^{\prime}$ in the $m$-moving frame as $t \rightarrow \infty$. The argument doesn't rely on the particular value of $y$, so in fact there exists $M(y, a)>0$ such that

$$
z(t, x+m(t) ; y, a)=\partial_{y} u(t, x+m(t) ; y, a) \rightarrow-M(y, a) U^{\prime}(x-s(y, a)) \quad \text { as } t \rightarrow \infty
$$

uniformly in $x \in \mathbb{R}$.

For the moment, let us fix $x$ and view $u(t, x+m(t) ; y, a)$ as a sequence of functions of $y$ indexed by time $t$. By (B.5), the sequence $u$ converges pointwise in $y$ as $t \rightarrow \infty$ to the limit $U(x-s(y, a))$. By (B.6), the derivative $\partial_{y} u$ converges pointwise in $y$ as $t \rightarrow \infty$ to the limit $-M(y, a) U^{\prime}(x-s(y, a))$. We recall a standard result from real analysis: if the convergence (B.6) is in fact uniform in $y \in(-1,1)$, then the limit of $u$ is differentiable in $y$ and its derivative is the limit of $\partial_{y} u$. Assuming this holds, we obtain

$$
\begin{aligned}
\lim _{t \rightarrow \infty} z(t, x+m(t) ; y, a) & =-M(y, a) U^{\prime}(x-s(y, a)) \\
& =\partial_{y}[U(x-s(y, a))]=-\partial_{y} s(y, a) U(x-s(y, a)) .
\end{aligned}
$$

Here, we have used the fact that $U(x-s(y, a))$ is differentiable in $y$ if and only if $s$ is. Taking $y=0$ in (B.7) and using the $x$-uniformity in (B.6), we obtain (B.1). Thus, it suffices to show that (B.6) holds uniformly in $y$ for each $x \in \mathbb{R}$ and $a>0$. 
To establish uniformity in $y$, we rely on a quantitative form of (B.6). The main result of [18] shows that the error in (1.14) is of order $t^{-1 / 2} ; C f$. (4.17). As mentioned earlier, (B.4) is structurally similar to the Fisher-KPP equation (1.12): after an appropriate shift and exponential tilt, both resemble the Dirichlet heat equation on $\mathbb{R}_{+}$. The argument of [18] only requires this general structure, so a straightforward adaptation of Theorem 1.3 in [18] implies

$$
z(t, x+m(t) ; y, a)=-M(y, a) U^{\prime}(x-s(y, a))+R(t, x ; y, a)
$$

for a remainder $R$ satisfying

$$
|R(t, x ; y, a)| \leq C(a) e^{-c|x|}(t+1)^{-1 / 2} .
$$

Crucially, this bound is independent of $y$. This is because $z$ only depends on $y$ through its initial data and the potential $f^{\prime}(u(t, x ; y, a))$ in (B.4). Now, the initial conditions $z(0, x ; y, a)=\delta(x-y+a)$ are supported in a common compact interval $[-a-1,-a+1]$ and have the same mass. Likewise, the potentials $f^{\prime}(u(t, x ; y, a))$ are monotone in $y$, and are thus controlled by the endpoints $y= \pm 1$. In particular, they are uniformly positive on the left and decay uniformly exponentially on the right. In this sense, they approximate the Dirichlet heat equation on $\mathbb{R}_{+}$uniformly-well in $y$.

The estimates in [18] are easily seen to be uniform in initial data and potentials of this form, so the error estimate (B.8) is independent of $y$. It follows that (B.6) holds uniformly in $y$ for fixed $x$. As noted above, the lemma follows.

\section{References}

[1] E. Aïdékon, J. Berestycki, É. Brunet, Z. Shi, Branching Brownian motion seen from its tip, Probab. Theory Relat. Fields 157, 2013, 405-451. 4

[2] L.-P. Arguin, A. Bovier, and N. Kistler, Poissonian statistics in the extremal process of branching Brownian motion, Ann. Appl. Probab. 22, 2012, 1693-1711. 4

[3] L.-P. Arguin, A. Bovier, and N. Kistler, The extremal process of branching Brownian motion, Probab. Theory Relat. Fields 157, 2013, 535-574. 4

[4] M. D. Bramson, Maximal displacement of branching Brownian motion, Comm. Pure Appl. Math. 31, 1978, 531-581. 2, 13

[5] M. D. Bramson, Convergence of solutions of the Kolmogorov equation to travelling waves, Mem. Amer. Math. Soc. 44, 1983. 2, 13

[6] É. Brunet and B. Derrida. Statistics at the tip of a branching random walk and the delay of traveling waves, Eur. Phys. Lett. 87, 2009, 60010.

[7] É. Brunet and B. Derrida. A branching random walk seen from the tip, Jour. Stat. Phys. 143, 2011, 420-446. 4, 8

[8] A. Cortines, L. Hartung, and O. Louidor, The structure of extreme level sets in branching Brownian motion, Ann. Probab. 47(4), 2019, 2257-2302. 4

[9] R. A. Fisher, The wave of advance of advantageous genes, Ann. Eugen. 7, 1937, 355-369. 2 
[10] C. Graham, Precise asymptotics for Fisher-KPP fronts, Nonlinearity 32, 2019, 1967-1998. 2, 18

[11] F. Hamel, J. Nolen, J.-M. Roquejoffre and L. Ryzhik, A short proof of the logarithmic Bramson correction in Fisher-KPP equations, Netw. Heterog. Media 8, 2013, 275-289. 2, 6, 9, 26, 35

[12] A. N. Kolmogorov, I. G. Petrovskii and N. S. Piskunov, Étude de l'équation de la diffusion avec croissance de la quantité de matière et son application à un problème biologique, Bull. Univ. État Moscou, Sér. Inter. A 1, 1937, 1-26. 2, 13

[13] S. P. Lalley and T. Sellke, A conditional limit theorem for the frontier of a branching Brownian motion. Annals of Probability 15, 1987, 1052-1061. 2

[14] K.-S. Lau, On the nonlinear diffusion equation of Kolmogorov, Petrovskii and Piskunov, J. Diff. Eqs. 59, 1985, 44-70. 2

[15] H. P. McKean, Application of Brownian motion to the equation of Kolmogorov-PetrovskiiPiskunov, Comm. Pure Appl. Math. 28 1975, 323-331. 2, 8

[16] L. Mytnik, J.-M. Roquejoffre and L. Ryzhik, Fisher-KPP equation with small data and the extremal process of branching Brownian motion, arXiv e-prints, 2020, 2009.02042. 4, 8

[17] J. Nolen, J.-M. Roquejoffre and L. Ryzhik, Convergence to a single wave in the Fisher-KPP equation, Chin. Ann. Math. Ser. B 38, 2017, 629-646. 2, 6, 9, 18, 35

[18] J. Nolen, J.-M. Roquejoffre and L. Ryzhik, Refined long-time asymptotics for Fisher-KPP fronts, Comm. Contemp. Math., 2018, 1850072. 2, 18, 39

[19] M. Roberts, A simple path to asymptotics for the frontier of a branching Brownian motion, Ann. Prob. 41, 2013, 3518-3541. 2

[20] K. Uchiyama, The behavior of solutions of some nonlinear diffusion equations for large time, J. Math. Kyoto Univ. 18, 1978, 453-508. 2 